\title{
Birth-and-Death Evolution and Reticulation of ITS Segments of Metschnikowia andauensis and Metschnikowia fructicola rDNA Repeats
}

\section{OPEN ACCESS}

Edited by:

Giovanna Suzzi,

Università di Teramo, Italy

Reviewed by:

Warren Albertin,

Institut des Sciences de la Vigne et du

Vin (ISW), France

Nicola Francesca,

Università degli Studi di Palermo, Italy

Pietro Buzzini,

University of Perugia, Italy

${ }^{*}$ Correspondence: Matthias Sipiczki

gecela@post.sk;

lipovy@tigris.unideb.hu

tPresent address:

Walter P. Pfliegler,

Department of Biotechnology

and Microbiology, University of Debrecen, Debrecen, Hungary

Specialty section:

This article was submitted to

Food Microbiology,

a section of the journal

Frontiers in Microbiology

Received: 12 March 2018

Accepted: 16 May 2018

Published: 12 June 2018

Citation:

Sipiczki M, Horvath E and

Pfliegler WP (2018) Birth-and-Death

Evolution and Reticulation of ITS

Segments of Metschnikowia andauensis and Metschnikowia

fructicola rDNA Repeats.

Front. Microbiol. 9:1193.

doi: 10.3389/fmicb.2018.01193

\section{Matthias Sipiczki*, Eniko Horvath and Walter P. Pflieglert \\ Department of Genetics and Applied Microbiology, University of Debrecen, Debrecen, Hungary}

The internal transcribed spacer (ITS) region (ITS1, 5.8S rDNA, and ITS2) separates the genes coding for the SSU $18 S$ and the LSU $26 S$ genes in the rDNA units which are organized into long tandem arrays in the overwhelming majority of fungi. As members of a multigenic family, these units are subject of concerted evolution, which homogenizes their sequences. Exceptions have been observed in certain groups of plants and in a few fungal species. In our previous study we described exceptionally high degree of sequence diversity in the D1/D2 domains of two pulcherrimin-producing Metschnikowia (Saccharomycotina) species which appeared to evolve by reticulation. The major goals of this study were the examination of the diversity of the ITS segments and their evolution. We show that the ITS sequences of these species are not homogenized either, differ from each other by up to 38 substitutions and indels which have dramatic effects on the predicted secondary structures of the transcripts. The high intragenomic diversity makes the D1/D2 domains and the ITS spacers unsuitable for barcoding of these species and therefore the taxonomic position of strains previously assigned to them needs revision. By analyzing the genome sequence of the $M$. fructicola type strain, we also show that the rDNA of this species is fragmented, contains pseudogenes and thus evolves by the birth-and-death mechanism rather than by homogenisation, which is unusual in yeasts. The results of the network analysis of the sequences further indicate that the ITS regions are also involved in reticulation. $M$. andauensis and $M$. fructicola can form interspecies hybrids and their hybrids segregate, providing thus possibilities for reticulation of the rDNA repeats.

Keywords: ribosomal RNA, ITS, evolution, pseudogene, reticulation, hybridisation, yeast, structure prediction

\section{INTRODUCTION}

The internal transcribed spacers ITS1 and ITS2 of the ribosomal DNA (rDNA) cistrons separate genes coding for ribosomal RNAs which are essential components of ribosomes. The rDNA cistrons are repeated many times in the genome accross the tree of life, so that enough rRNA can be produced when demand for ribosomes is high. In most species for which relevant molecular data are available, the rDNA cistrons flanked by characteristic intergenic regions are organized 
as tandem head-to-tail repeats (rDNA repeats) in continuous arrays (for a review see Torres-Machorro et al., 2010). In spite of the large number of repeats, nucleotide polymorphism within the rDNA array is usually very low (e.g., Ganley and Kobayashi, 2007). By a process called "concerted evolution" (Zimmer et al., 1980; Elder and Turner, 1995), the repeats within the arrays are maintained essentially identical (homogenized) even over evolutionarily significant timescales. It ensures that a mutation that arises in one repeat is either eliminated or spreads by intercopy interactions through the array until fixation.

The ITS spacers are transcribed together with the rRNA genes of the cistron but their RNA copies are not incorporated into mature ribosomes, but removed from the ribosomal precursor RNA (prerRNA) by a specific cleavage process that is catalyzed by the secondary structures of the ITS transcripts themselves (for reviews, see Venema and Tollervey, 1999; Coleman, 2015) (Figure 1). Despite this activity, these sequences are assumed to evolve neutrally (Schlotterer et al., 1994) and thus considered suitable as genomic markers for taxonomic identification and phylogenetic reconstructions (Hillis and Dixon, 1991). Since their sequence similarity is greater within a species than between species (barcoding gap), the ITS region was recently designated the official barcode for fungi (Schoch et al., 2012). In our previous work (Sipiczki et al., 2013) we presented data indicating that the rDNA homogenisation mechanism is not always effective. We demonstrated that two Metschnikowia species had nonhomogenized pools of D1/D2 domains of LSU (26S) rRNA genes.

The ascomycetous yeast genus Metschnikowia consists of over 80 species (Lachance, 2016). The small-spored M. pulcherrima and five related species ( $M$. andauensis, $M$. fructicola, $M$. shanxiensis, $M$. sinensis, and $M$. ziziphicola) produce pulcherrimin, a red-brownish pigment in their cells and also externally, in the medium, observable as halos around the colonies growing on agar media (e.g., Kluyver et al., 1953; Sipiczki, 2006; Xue et al., 2006; Lachance, 2011). Pulcherrimin is a water-insoluble complex of $\mathrm{Fe}^{3+}$ ions and the diffusible pulcherriminic acid (a derivative of the cyclo-L-leucyl-L-leucyl dipeptide) (Cook and Slater, 1956; Cryle et al., 2010). The

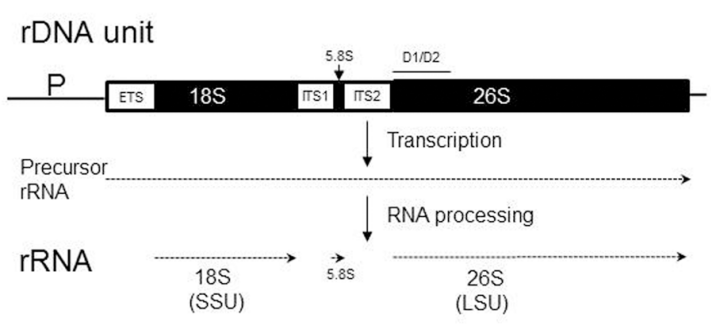

FIGURE 1 | Organization and transcription of the chromosomal rDNA unit (repeat) containing ribosomal RNA (rRNA) genes. The three rRNA genes (18S, 5.8S, and 26S) are shown as solid boxes, while regions removed from the precursor transcript during RNA maturation (ETS, external transcribed spacer; ITS, internal transcribed spacer) are open boxes. The 18S rRNA is integrated into the small subunit (SSU) of the ribosome, whereas the $5.8 \mathrm{~S}$ and $26 \mathrm{~S}$ rRNAs are embedded into the large subunit (LSU). P, promoter. The position of the D1/D2 domain is shown by the line over the 26S rRNA gene. depletion of the medium of free $\mathrm{Fe}^{3+}$ by the formation of the pigment has inhibitory effect on the germination of fungal conidia and the growth of certain molds, yeasts and bacteria (Sipiczki, 2006). A recent phylogenetic analysis based on data from four protein-coding genes placed the pulcherrimineproducing species on a lineage clearly separated from the rest of the genus (Guzmán et al., 2013).

We found that the type strains of $M$. andauensis and M. fructicola have non-homogenized rDNA arrays (Sipiczki et al., 2013). The intragenomic diversity of the D1/D2 domains of the large subunit (LSU; 26S) rDNA (Figure 1) hampers the taxonomic separation of them from each other and from related species by D1/D2 sequencing. Both strains turned out to have D1/D2 units of diverse sequences that do not form distinct clades on the phylogenetic trees and networks and appear to evolve by reticulation including interspecies interactions. The aims of this study were to examine the diversity of ITS segments of the rDNA repeats of these species by cloning and sequencing individual paralogs, the identification of ITS sequences in the draft genome sequence of the $M$. fructicola type strain and their phylogenetic analysis. We show that the ITS sequences of $M$. andauensis and M. fructicola are not homogenized either and are likewise placed intermixed in the phylogenetic networks. Hence, neither D1/D1 nor ITS sequencing can be used for barcoding of these species. By analyzing the genome sequence of the $M$. fructicola type strain, we also show that the rDNA of this species is fragmented, contains pseudogenes and thus evolves by the birth and death mechanism rather than by homogenisation. In fungi, birth-anddeath mode of rDNA evolution was observed only in the case of dispersed 5S genes (Rooney and Ward, 2005) which, however, have no relevance for barcoding and taxonomic identification. We also present data demonstrating that both species can form interspecies hybrids and the hybrids segregate, providing thus possibilities for reticulation of their rDNA repeats with those of related species.

\section{MATERIALS AND METHODS}

\section{Strains and Culture Conditions}

The $M$. andauensis and M. fructicola strains used are listed in Supplementary Table S1. The composition of the growth media YEA (yeast-extract agar), YEL (yeast-extract liquid), and SMA (synthetic minimal agar), were described previously (Sipiczki, 2012). Sporulation was tested on V-8 agar (Pitt and Miller, 1968) and vegetable juice (BIO Gemüsesaft, Josef Pölz, Bio-Produkte, 84518 Garching an der Alz, Germany) agar. V8 juice was filtered with filter paper, diluted 1:20 with distilled water, adjusted to $\mathrm{pH}$ 5.5 by $\mathrm{NaOH}$, and the medium thus prepared autoclaved. The vegetable juice was used in $20 \mathrm{x}$ dilution without $\mathrm{pH}$ adjustment. Both media contained $2 \%$ agar.

\section{Amplification, Cloning, and Sequencing of ITS1-5.8S-ITS2}

Nuclear DNA was isolated from overnight cultures grown in YEL broth as described previously (Sipiczki, 2003). The isolated DNA was used for the amplification of the ITS1-5.8S-ITS2 
regions of the rRNA repeats with the primers ITS1 and ITS4 (White et al., 1990) and GoTaq polymerase (Promega, Madison, United States). The amplified DNA was used either for direct sequencing or for random cloning of individual ITS1-5.8SITS2 fragments into the pGEM-T Easy Vector, following the manufacturer's instructions (Promega, Madison, United States). In the latter case, bacterial colonies were randomly selected from the transformants. Plasmids were extracted from the colonies and checked for the size of the inserts by reamplification with the same primer pair. The amplified DNA was sequenced in both directions using the same primers. The sequences were deposited in GenBank under accession numbers listed in Supplementary Table S2. The cloned sequences were compared with each other using the bl2seq algorithm available in $\mathrm{NCBI}^{1}$. Search for similar database sequences was conducted using the blastn services of the NCBI Blast web site and the UNITE database 2 .

\section{Search for ITS Sequences in the M. fructicola Genome Sequence}

The only sequence found in more than one clones was used as query sequence to find complete and partial ITS1-5.8S-ITS2 segments in the draft genome sequence of CBS $8853^{\mathrm{T}}$, the type strain of $M$. fructicola available under the accession number ANFW00000000.2 in the NCBI Genome database ${ }^{3}$. The identified rDNA units were checked for completeness by the examination of their components, including the sequences of the genes coding for the 18S SSU (small subunit) and the 26S LSU (large subunit) rRNAs. All genomic ITS1-5.8S-ITS2 sequences were compared with each other and with the cloned sequences using bl2seq (see above).

\section{RNA Secondary Structure Prediction}

ITS1 secondary structures were predicted from nucleotide sequences with the algorithm available at the mfold Web Server ${ }^{4}$ (Zuker, 2003). The delta $G$ required for the formation of the secondary structures shown in relevant figure were -1.03 (Ifr9), -2.11 (Ifrc26), -3.10 (Ian10), -4.50 (Ifrc4), and -6.70 (Ian51). For the ITS2 consensus secondary structure prediction, the ITS2 database pipeline ${ }^{5}$ (Ankenbrand et al., 2015) was used. This workbench determines complete individual secondary structures for ITS2 sequences based on energy minimization (Markham and Zuker, 2008) or iterative homology modeling (Wolf et al., 2005). The secondary structures were re-drawn for publication purposes using the forna package http://rna.tbi.univie.ac.at/ forna/ (Kerpedjiev et al., 2015). RNA weblogo was created using https://rth.dk/resources/slogo/ (Gorodkin et al., 1997).

\section{Phylogenetic and Network Analysis}

To obtain multiple alignments for phylogenetic analyses, the sequences were aligned with the Clustal W 1.7 (Thompson et al., 1994) algorithm. Statistical parsimony networks were

\footnotetext{
${ }^{1}$ http://blast.ncbi.nlm.nih.gov/Blast.cgi

${ }^{2}$ https://unite.ut.ee/analysis.php

${ }^{3}$ https://www.ncbi.nlm.nih.gov/genome/

${ }^{4}$ http://unafold.rna.albany.edu/?q=mfold

${ }^{5}$ http://its2.bioapps.biozentrum.uni-wuerzburg.de/
}

constructed with TCS 1.21, a program that implements the estimation of genealogies of DNA sequences from their multiple alignment (Clement et al., 2000). The default 95\% cutoff was used. From the same Clustal alignments rooted rectangular phylogenetic networks and neighbor-net splits graphs were also created with the SplitsTree4 V4.12.8 package (Bryant and Moulton, 2004). In these analyses, the DNA evolution model HKY 85 (Hasegawa et al., 1985) was used for distance calculation. The outgroup of the rectangular phylogram was the sequence FJ623593 of $M$. chrysoperlae CBS $9803^{\mathrm{T}}$. To generate neighbornets, Equal Angle setting was chosen and the sequence used as outgroup on the rectangular phylogram was excluded from the analysis, as the neighbor nets are unrooted networks. To test the aligned sequences for recombination, we used the Phi test of Bruen et al. (2006) as available in the Splits Tree4 package.

\section{Examination of Sporulation and Spore Germination}

To induce sporulation, dense suspensions of overnight YPGL cultures were dropped on V-8 agar and vegetable juice agar. Sporulation was examined microscopically after incubation at $17^{\circ} \mathrm{C}$ at regular time intervals for 4 weeks. To visualize the nuclei of the ascospores, samples of the sporulating cultures were suspended in $40 \%$ ethanol, stained with $4^{\prime}, 6$-diamidino2-phenylindole (DAPI) (Sipiczki et al., 1998) and examined in a fluorescence microscope. Spore germination was examined microscopically in asci pulled out from dense suspensions of sporulating cultures with a micromanipulator on the surface of YEA plates and in samples of the cultures spread on thin YEA films prepared on glass slides and covered with cover slips. The latter ("sandwich") method originally developed for the examination of morphological transitions in yeast cultures was described previously (Sipiczki et al., 1998). The incubation temperature was $20^{\circ} \mathrm{C}$. Microphotographs were taken with an Olympus BX51 microscope and a DP70 digital camera. As the asci remained intact on the complete medium even after 4 weeks of incubation, we assumed that the failure of the ascus wall dissolution might prevent the spores from germination. Therefore we performed the experiments also with ascospores released from asci by Zymolyase 20T (MP Biomedicals, California, United States) treatment $(0.5-1.0 \mathrm{mg} / \mathrm{ml}$ $\mu \mathrm{g} \mathrm{ml}^{-1}, 25^{\circ} \mathrm{C}, 3 \mathrm{~h}$ ). To test the effect of freezing shock on germination activity, samples frozen to $-20^{\circ} \mathrm{C}$ for 10 days before spreading on the test medium were also examined.

\section{Mutagenesis and Characterisation of Mutants}

Cells of overnight cultures grown in YEL broth at room temperature were mutagenised either by UV irradiation on YEA plates or by culturing in YEL supplemented with nitrosoguanidine. For UV mutagenesis $10^{3}$ cells were spread on the plates and irradiated for time periods required to kill $85-90 \%$ of cells. After 7 days of incubation at $30^{\circ} \mathrm{C}$, the colonies produced by the surviving cells were replica-plated onto SMA plates to identify auxotrophic mutants. The colonies that did not grow on SMA were isolated for physiology tests. To 
induce mutations by nitrosoguanidine, the $0.5-\mathrm{ml}$ samples of the overnight culture $\left(\sim 10^{8}\right.$ cells $\left.\mathrm{ml}^{-1}\right)$ were added to $0.5 \mathrm{ml}$ of fresh YEL supplemented with various amounts of nitrosoguanidine and incubated at room temperature for $1 \mathrm{~h}$ to allow DNA synthesis in the presence of the mutagen. The mutagenised cultures were then diluted with sterile water to $\sim 10^{4}$ cells $\mathrm{ml}^{-1}$ and 100- $\mu$ l aliquots were spread on YEA plates. The colonies of plates showing 10-20\% survival were replica-plated after 7 days of incubation onto SMA plates. The colonies whose replicas did not grow on SMA were isolated as putative auxotrophic mutants.

The nutritional requirements of the mutants were determined by the two-step disk method described previously (Sipiczki and Ferenczy, 1978). Briefly, two SMA plates were seeded with dense suspensions of cells of the mutant. Then six sterile filter-paper disks, each dipped into a different solution of 5-6 amino acids and nucleotides were placed in a hexagonal pattern on the surface of one plate. After 3 days of incubation, a zone of growth appeared around the disk that contained the compound for which the mutant was auxotrophic. Next, disks soaked separately with solutions of the individual members of the positive group were placed on the second plate. After 3 days of incubation, growth was visible around the disk soaked with the amino acid or nucleotide base which could not be synthesized in the mutant cells. The mutants are listed in Supplementary Table S1. The mutants used successfully in the hybridisation experiments have been deposited in the CBS culture collection (Centraalbureau voor Schimmelcultures, Utrecht, Netherlands).

\section{Hybridisation and Segregation Analysis}

The auxotrophic mutants to be hybridized were streaked on YEA plates (two strains on one plate). After 3 days of incubation the line-shaped cultures were replica-plated onto fresh plates perpendicularly to each other to produce grids of prints in which the mutants intersected each other. After 7 days of incubation at $20^{\circ} \mathrm{C}$, the "grids" were replica-plated onto SMA plates on which the mutants could not grow. If hybridization took place, prototrophic colonies appeared at the intersections. Individual colonies were isolated from the intersections and inoculated onto fresh SMA plates to obtain pure hybrid cultures.

To examine segregation, the hybrids were inoculated on vegetable agar plates and incubated at $13-15^{\circ} \mathrm{C}$. After 4 weeks of incubation samples were taken from the cultures and spread on YEA plates (1) directly or (2) after Zymolyase treatment which lysed most vegetative cells and freed the ascospores from the asci or (3) after 10 days of freezing $\left(-20^{\circ} \mathrm{C}\right)$. The colonies produced were replica-plated on SMA plates to identify auxotrophic segregants. The colonies that did not grow on SMA were isolated from the plates and tested for auxotrophies by inoculating them onto SMA plates supplemented with compounds for which the parental strains were auxotrophic.

\section{RESULTS}

\section{Diversity of Cloned ITS Sequences}

When the DNA amplified with the ITS1 and ITS4 primers from the type strains was directly sequenced with the ITS1 primer, the chromatograms were not readable in the segments corresponding to the ITS1 spacers, and the sequences of the $5.8 \mathrm{~S}$ and ITS 2 parts contained about $20 \%$ ambiguous nucleotides. When sequenced from the ITS4 primer, the latter two segments had only a few ambiguous positions but ITS1 was chaotic again. These results implied that the ITS regions of the rDNA units like their D1/D2 domains (Sipiczki et al., 2013) were heterogeneous in both strains and thus unsuitable for their taxonomic differentiation. To verify the heterogeneity, individual molecules ( 6 from the $M$. andauensis type strain and 7 from the $M$. fructicola type strain) were cloned from the amplified DNA and sequenced individually. None of the cloned sequences had ambiguous positions but they turned out to differ from each other at numerous positions (Table 1). Both the ITS1 and ITS2 segments were much shorter than those of $S$. cerevisiae S288c $c^{6}$ and comparable in size to those of other Metschnikowia species. All but two ITS1 segments consisted of 74 nucleotides; the exceptions were Ian10 (73 nt) and Ian51 (76 nt). All ITS2 segments of both strains were 110 nucleotide long. 5 out of the $8 \mathrm{M}$. fructicola clones had identical sequences (Ifr4, Ifr6, Ifrb13, Ifrb15, and Ifrb17), whereas all M. andauensis clones had unique sequences. The most different sequences were $M$ andauensis Ian51 and M. fructicola Ifrc26, but their difference (26 substitutions and 1 indel) was only slightly bigger than the biggest intraspecies differences (26 in M. andauensis) and (24 in M. fructicola).

ITS1 was more diverse than ITS2. In the Clustal alignment of all cloned sequences 34 positions were variable in the ITS1 segments and four positions showed polymorphism in the ITS2 segments. Five $M$. fructicola clones and one $M$. andauensis clone had identical ITS2 sequences. In the $5.8 \mathrm{~S}$ genes only one site was polymorphic. At this position all $M$. andauensis and two $M$. fructicola sequences had a cytosine whereas the rest of the $M$. fructicola sequences had a thymine. Interestingly, the range of intragenomic differences was broader in both strains than the differences of certain cloned sequences from the ITS sequences of the type strains of certain related species. For example Ian 10 differed from Ian51 at 26 positions but only at 12 positions from KY104196 of $M$. chrysoperlae CBS $9803^{\mathrm{T}}$. The ITS1 region of Ian51 differed at 10 sites from that of M. rubicola NRRL Y- $6064^{\mathrm{T}}$ (not released yet; Kurtzman et al., 2018) and at 14 positions from MF446617 of M. persimmonesis KIOM G15050 ${ }^{\mathrm{T}}$. The ITS1 region of Ifrb17 differed from that of Ifrc 26 by 24 substitutions but only by 3 substitutions from the corresponding sequence of $M$. leonuri NRRL Y-6546 ${ }^{\mathrm{T}}$ (not released yet; Kurtzman et al., 2018). The blast search in the UNITE database identified a M. bicuspidata var. bicuspidata sequence (KP132407) which differed by 3 and 6 substitutions from Ifrb17 and Ian51, respectively.

\section{ITS Regions in a $M$. fructicola Draft Genome Sequence}

Recently the draft genome sequence of the strain M. fructicola 277 has been deposited in the NCBI genome database (Hershkovitz et al., 2013). This strain is presumably identical with the type strain of the species maintained in culture collections under

${ }^{6}$ https://www.yeastgenome.org/search?q=ITS1\%20ITS2\&is_quick=true 
TABLE 1 | Number of differences in the pair-wise Blast alignments of the cloned sequences.

\begin{tabular}{|c|c|c|c|c|c|c|c|c|c|c|c|c|c|}
\hline & Ian5 & $\operatorname{lan} 6$ & lan10 & Ian42 & Ian43 & $\operatorname{lan} 51$ & Ifr4 & Ifr6 & Ifr9 & Ifrb13 & Ifrb15 & Ifrb17 & Ifc26 \\
\hline $\operatorname{lan} 6$ & 11 & & & & & & & & & & & & \\
\hline Ian10 & 21 & 22 & & & & & & & & & & & \\
\hline Ian42 & 6 & 10 & 25 & & & & & & & & & & \\
\hline Ian43 & 5 & 6 & 26 & 6 & & & & & & & & & \\
\hline lan51 & 11 & 4 & 26 & 8 & 6 & & & & & & & & \\
\hline Ifr4 & 11 & 7 & 23 & 7 & 11 & 7 & & & & & & & \\
\hline Ifr6 & 11 & 7 & 23 & 7 & 11 & 7 & 0 & & & & & & \\
\hline Ifr9 & 10 & 10 & 22 & 6 & 10 & 13 & 7 & 7 & & & & & \\
\hline Ifrb13 & 11 & 7 & 23 & 7 & 11 & 7 & 0 & 0 & 7 & & & & \\
\hline Ifrb15 & 11 & 7 & 23 & 7 & 11 & 7 & 0 & 0 & 7 & 0 & & & \\
\hline Ifrb17 & 11 & 7 & 23 & 7 & 11 & 7 & 0 & 0 & 7 & 0 & 0 & & \\
\hline Ifrc26 & 20 & 23 & 15 & 24 & 25 & 27 & 24 & 24 & 21 & 24 & 24 & 24 & \\
\hline Ifrc27 & 11 & 15 & 23 & 9 & 11 & 13 & 12 & 12 & 11 & 12 & 12 & 12 & 22 \\
\hline
\end{tabular}

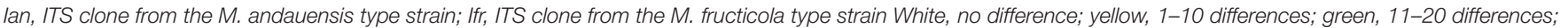
blue, 21-27 differences.

collection numbers NRRL Y-27328 ${ }^{\mathrm{T}}$ and CBS $8853^{\mathrm{T}}$ (Kurtzman and Droby, 2001). As we cloned the sequences from a CBS $8853^{\mathrm{T}}$ culture, we searched ANFW01000000 for sequences similar to the clones. The blast search identified 8 loci containing fulllength ITS1-5.8S-ITS2 regions (Table 2 and Figure 2) and 2 incomplete copies. The sequence of the 5 identical $M$. fructicola clones was found only in the locus identified in unitig 150. None of the other loci showed $100 \%$ identity with any of the cloned sequences. When compared with each other, only the two genomic loci of unitig 187 showed $100 \%$ sequence identity. The pairwise differences of the rest were comparable to those found between the cloned sequences. The truncate copies in unitigs 9 and 189 lacked the entire ITS1 segment and their ITS2 sequences had 6 polymorphic sites that were conserved in the cloned sequences. Their $5.8 \mathrm{~S}$ genes were very different from those in the cloned sequences and in the other genomic loci. The big difference between these truncate genomic regions and the cloned sequences shown in Table 2 are mainly due to substitutions in the $5.8 \mathrm{~S}$ genes.

Two unitigs contained pairs of complete rDNA units. In 5 unitigs the units were terminally located indicating that they can be terminal parts of genomic rDNA arrays, which the assembling program could not fully reconstruct. The unit in unitig 32 is far enough from both ends of the unitig to assume that it might be a solo copy. The truncate copies were also internally located in their unitigs but were not accompanied by $18 \mathrm{~S}$ genes and the $26 \mathrm{~S}$ genes behind their ITS2 sequences were incomplete.

The complete $26 \mathrm{~S}$ genes found in 8 loci offered a possibility to examine whether any of them had sequences identical with any of the D1/D2 domains cloned in our previous study (Sipiczki et al., 2013). Therefore we blasted the cloned D1/D2 sequences against ANFW01000000. Identity was found only between the M. fructicola D1/D2 clone fb6 (KC411964) and the 26S gene located in unitig 150. Remarkably, this rDNA unit had the only ITS sequence that was found also in certain cloned ITS segments. The rest of the genomic sequences differed by 1-11 and 2-15 substitutions from the D1/D2 clones of M. fructicola and $M$. andauensis, respectively. Similar search in M. andauensis could not be performed because its genome has not been sequenced yet.

\section{Phylogenetic Network Analysis}

Although we cannot exclude that certain genomic ITS segments could be sequence chimeras arising from misassembly of reads, we aligned them with the cloned sequences for phylogenetic network analyses. The statistical parsimony network analysis grouped most sequences of both type strains in a single network (Figure 3). Within the network, the sequences of the two strains did not form distinct lineages. This finding implies that the ITS sequences of the two strains might have joint evolutionary history, during which the rDNA units of the strains interacted many times. The program identified as ancestral the group of identical sequences cloned from $M$. fructicola. These sequences differed from certain $M$. andauensis members of the network by fewer substitutions than from certain other $M$. fructicola sequences. One $M$. andauensis clone, one $M$. fructicola clone and one genomic sequence were not connected to the network as if they had evolved from different ancestors.

The intermixing of the sequences of the two strains suggested that their ITS regions evolved in a reticulate way. To visualize reticulate events we generated rooted rectangular phylogenetic networks and neighbor-net splits graphs with SplitsTree4 V4.12.8 (Bryant and Moulton, 2004). In the rooted rectangular phylogenetic network shown in Figure 4, the network edges connecting nodes in a non-bifurcational way indicate interactions between lineages. The high number of network edges clearly demonstrate that reticulation have played a major role in the evolution of the ITS segments examined. The result of the neighbor-net network analysis is shown in Figure 5. In a splits graph, a pair of nodes may be linked by a single edge (tree-like part) or a set of parallel edges. The latter depict alternative evolutionary possibilities (reticulate part). The box-like structures confirmed the notion that reticulation has occurred in the evolution of these sequences indeed. Reticulation 


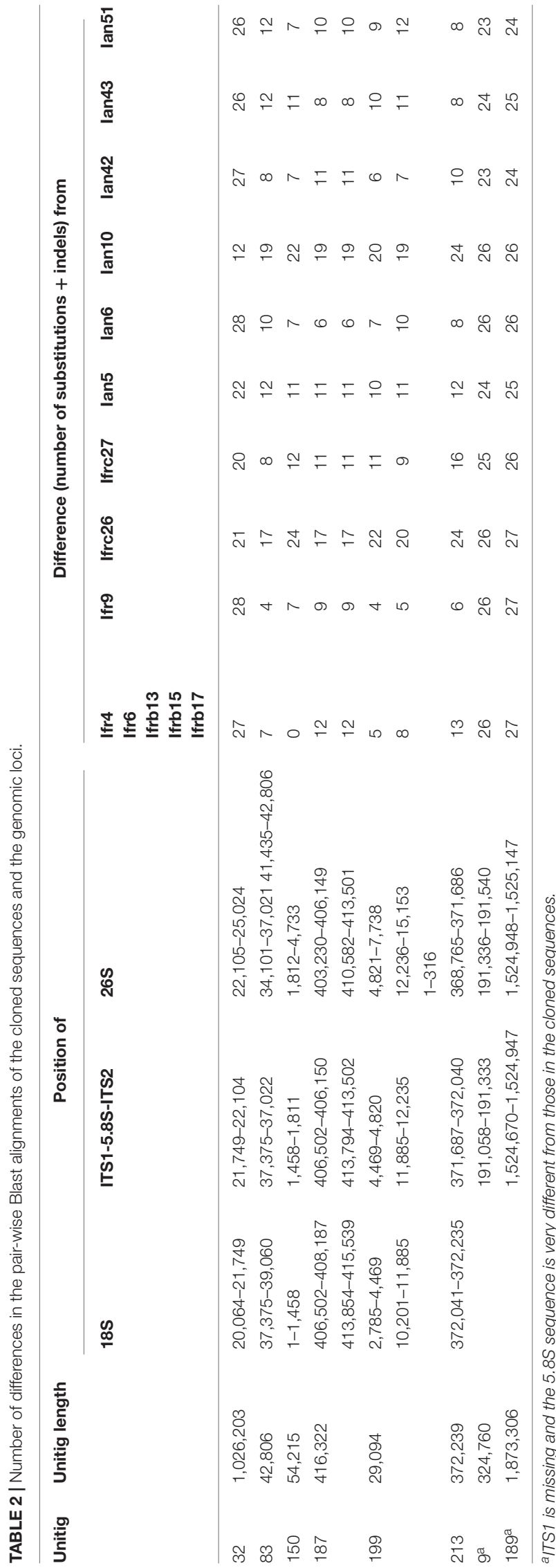

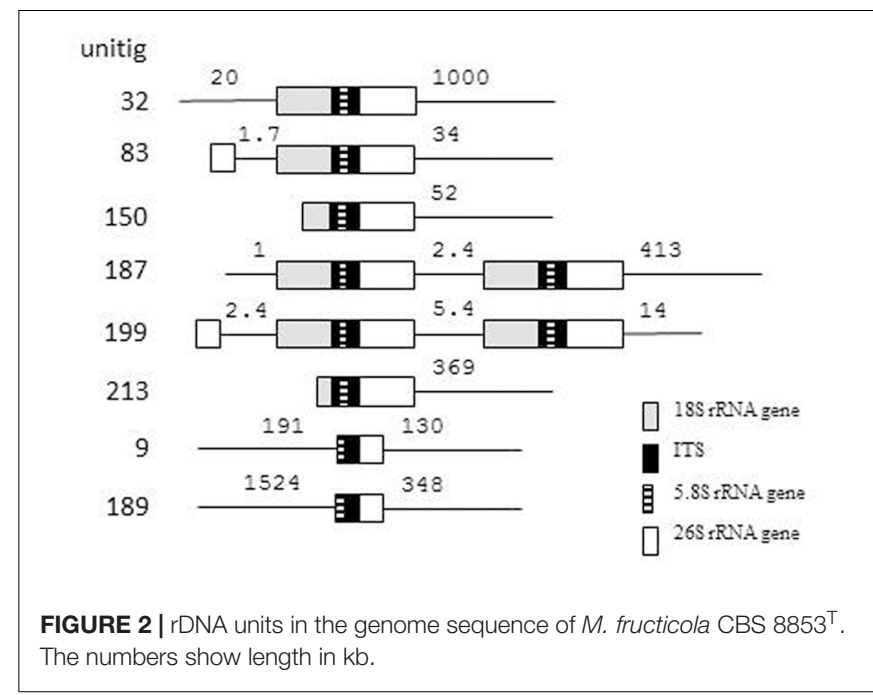

involves complex processes such as horizontal transfer and exchange of genetic elements, hybridisation and recombination. The Phi test (Bruen et al., 2006) found statistically significant evidence for recombination ( $p=2.784 \mathrm{E}-8)$.

\section{Location of the Variable Positions and the Effect of Substitutions on the Predicted Secondary Structures of the Transcripts}

The CLUSTAL alignment of all cloned sequences revealed that most nucleotides of the ITS1 and ITS2 regions were stable in all sequences, and the variable positions were not randomly scattered. To examine the possible structural effects of nucleotide differences in the variable positions, we generated secondary ITS1 and ITS2 rRNA structures from each cloned sequence. Secondary structures can provide information not found in the primary sequence (Caetano-Anolle's, 2002). The ITS1 structures were very diverse (examples are presented in Figure 6) showing limited structural conservatism. Most but not all sequences formed a hairpin with several bulges of diverse size. The tip loop of these hairpins contained a conserved a-a pair at sites 39-40. These nucleotides may be important for the folding of the RNA transcript because they are conserved in the tip loop of the folded S. cerevisiae ITS1 as well (Coleman, 2015). The other variable positions formed four groups, three of them located in the backfolding stretch of the loop stem. Changes of nucleotides in certain positions caused drastic changes in the secondary structures. The ITS2 sequences had only four variable sites and their predicted secondary structures were identical. All had four loops with helix III as the longest loop (Figure 7). This structure corresponds to the core structure deduced from the analysis of 5000 ITS2 sequences (Schultz et al., 2005). In contrast to the substitution of ITS1, the substitutions in the ITS2 sequences had no effect on the predicted secondary structure. All but one variable positions grouped in the unpaired segment located between the Large loop III and the small loop IV. The only exception was in the tip of loop II. 


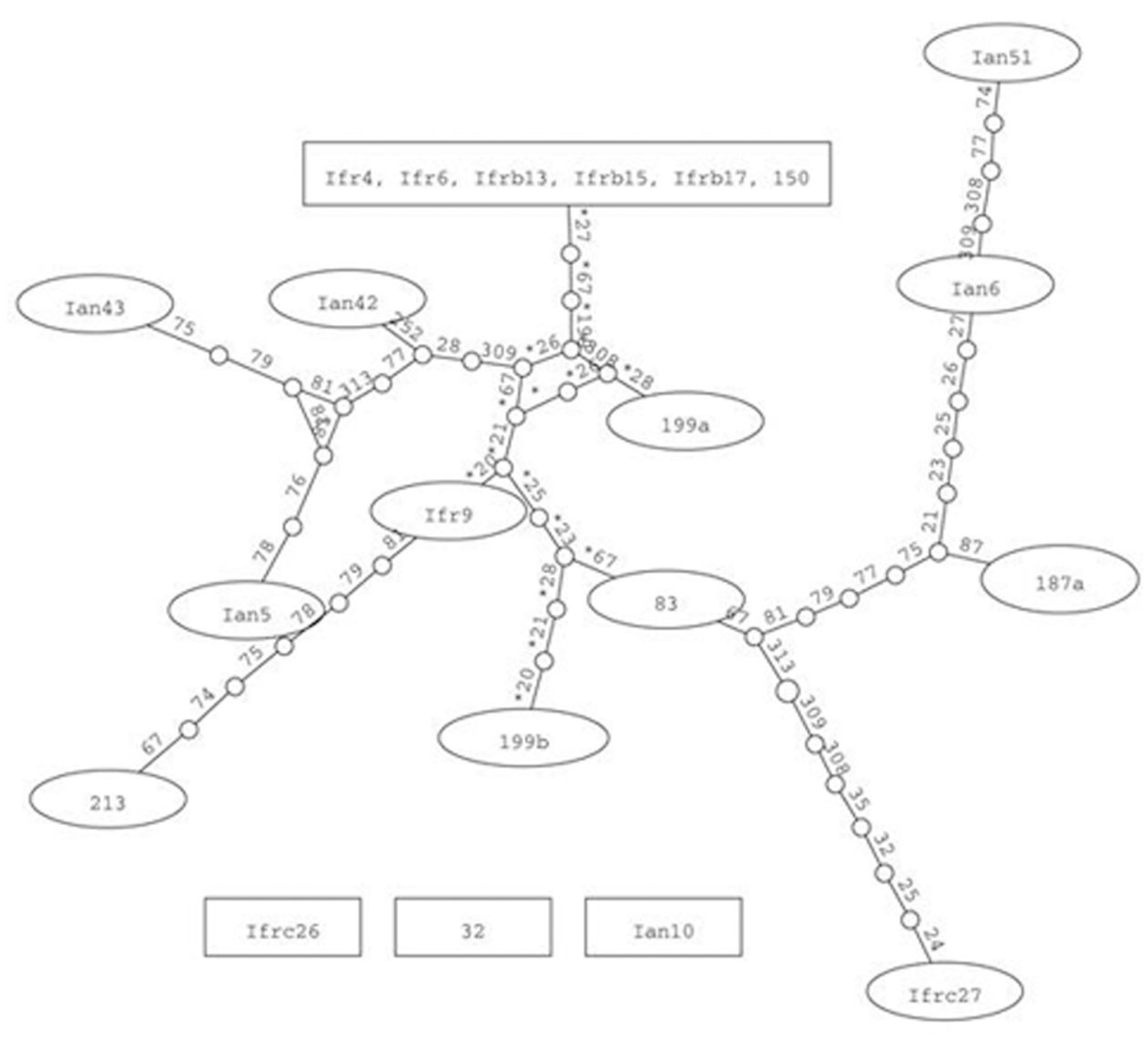

FIGURE 3 | Parsimony network analysis showing the genealogical relationships of the cloned ITS1-5.8S-ITS2 sequences. The connections are based on the set of plausible solutions with a 95\% of parsimony probability. Each connecting line represents one substitution and each small circle represents a missing intermediate sequence. The numerals between the circles are the serial numbers of nucleotides from the beginning of the alignment. A rectangle denotes the sequence identified as ancestral by the analysis. Ifr 4 represents the group of five $M$. fructicola clones of identical sequences. Three sequences were excluded from the network by the algorithm.

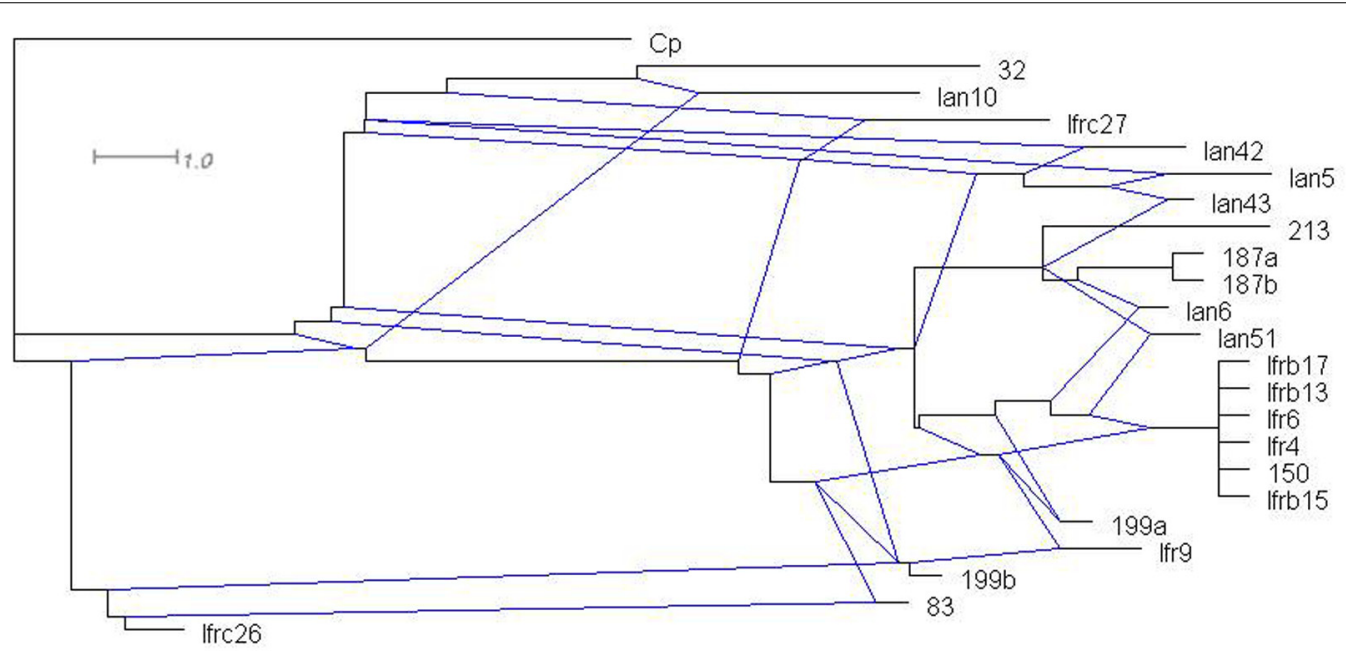

FIGURE 4 | Rooted rectangular phylogenetic network of the cloned sequences. Cp (C. picachoensis CBS 9804 ${ }^{\top}$ AY494780) is the outgroup. The scale bar represents the split support for the edges. 


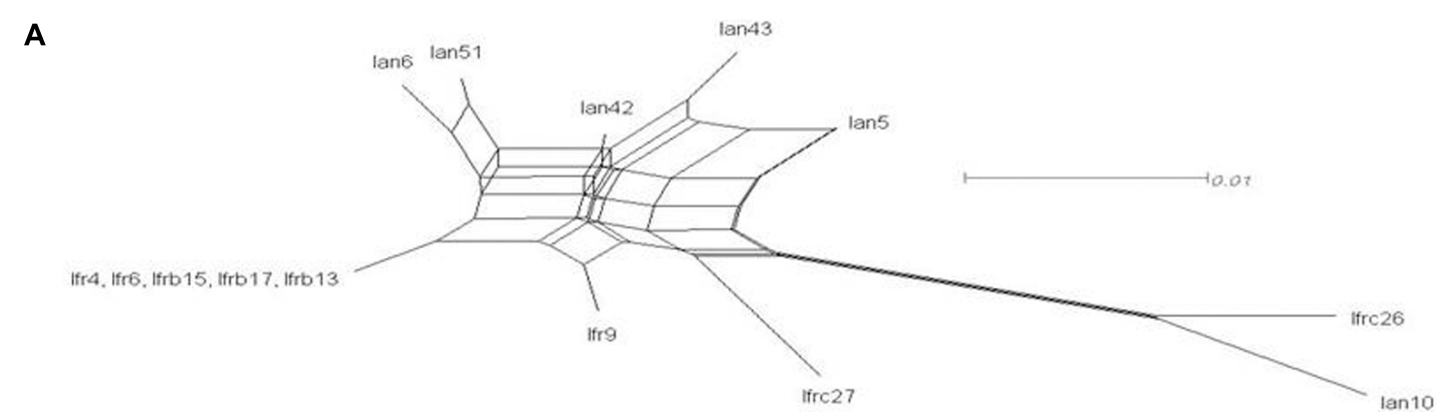

B

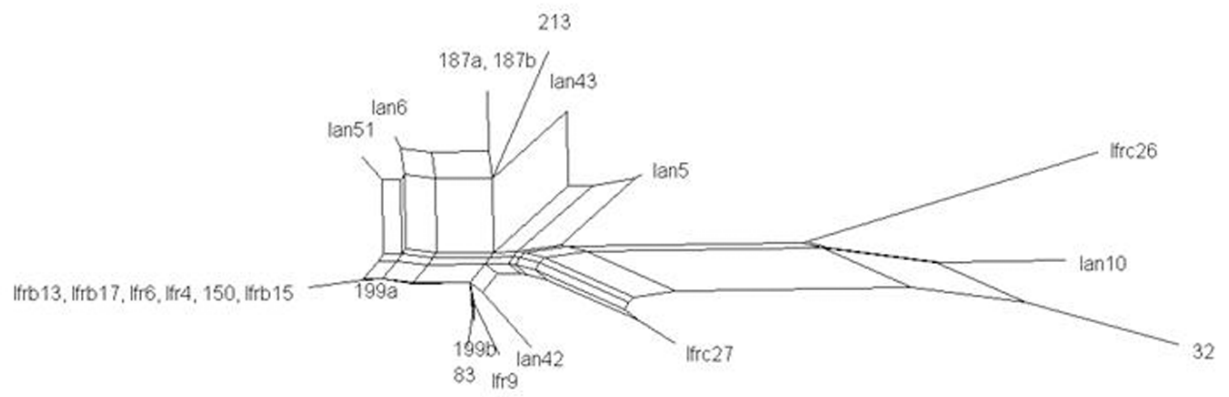

FIGURE 5 | Network analysis of the cloned ITS1-5.8S-ITS2 sequences. Neighbor-net splits graphs. (A) Cloned sequences. (B) Cloned and genomic sequences. The scale bar represents the split support for the edges.

\section{A}

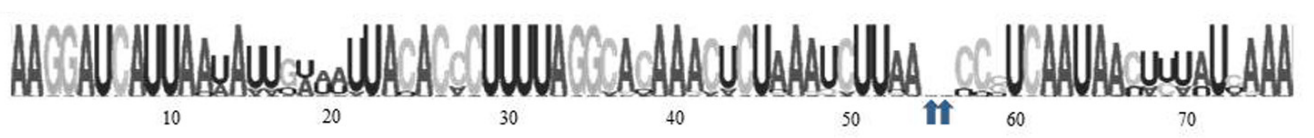

B

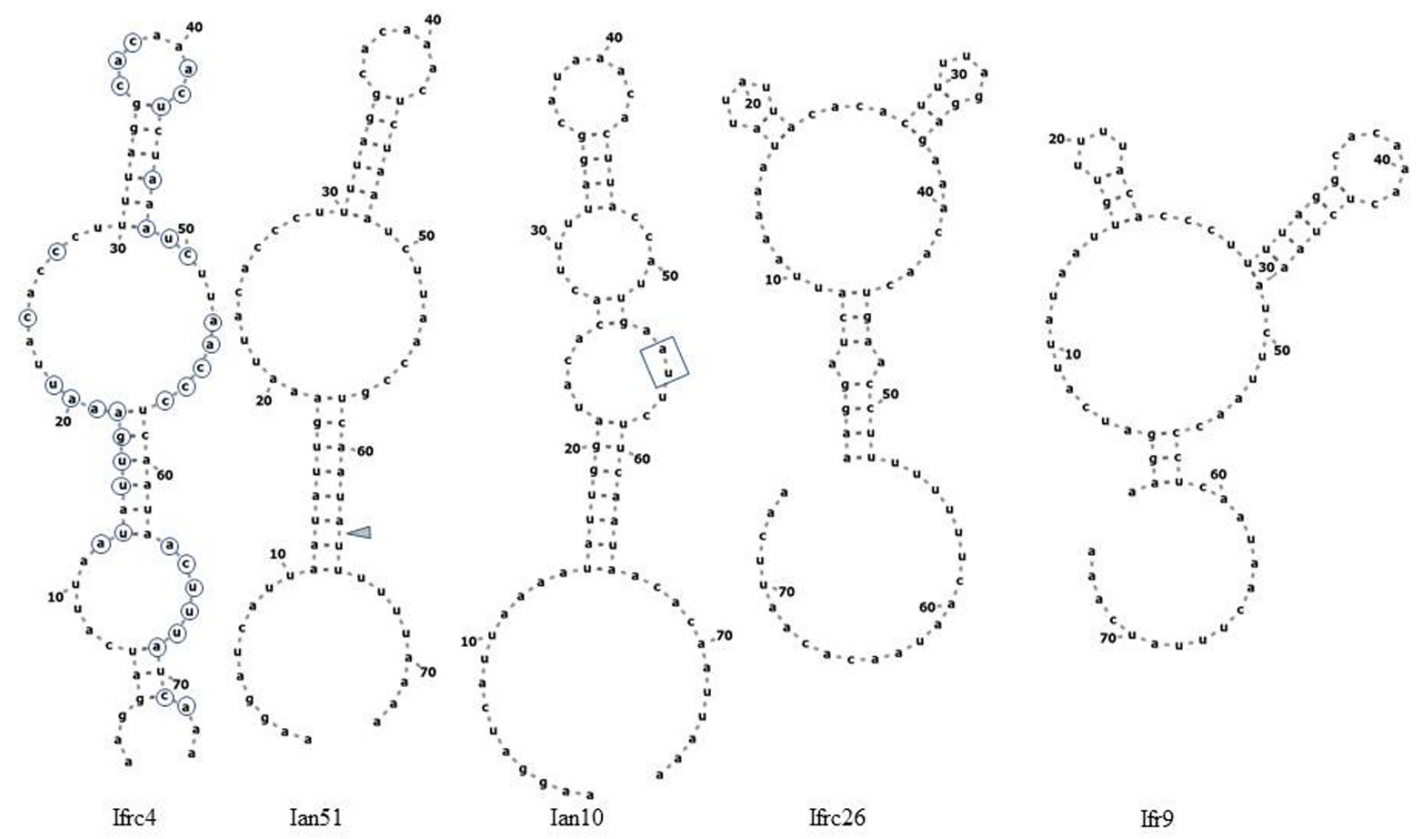

FIGURE 6 | Structural analysis of ITS1. (A) RNA weblogo. Arrowheads denote sites of insertions of two nucleotides in lan10. (B) Predicted secondary structures of cloned ITS1 sequences. The positions circled on Ifrc4 are variable. The insertions in lan10 are boxed. The gray triangle marks the site of deletion in lan51. 


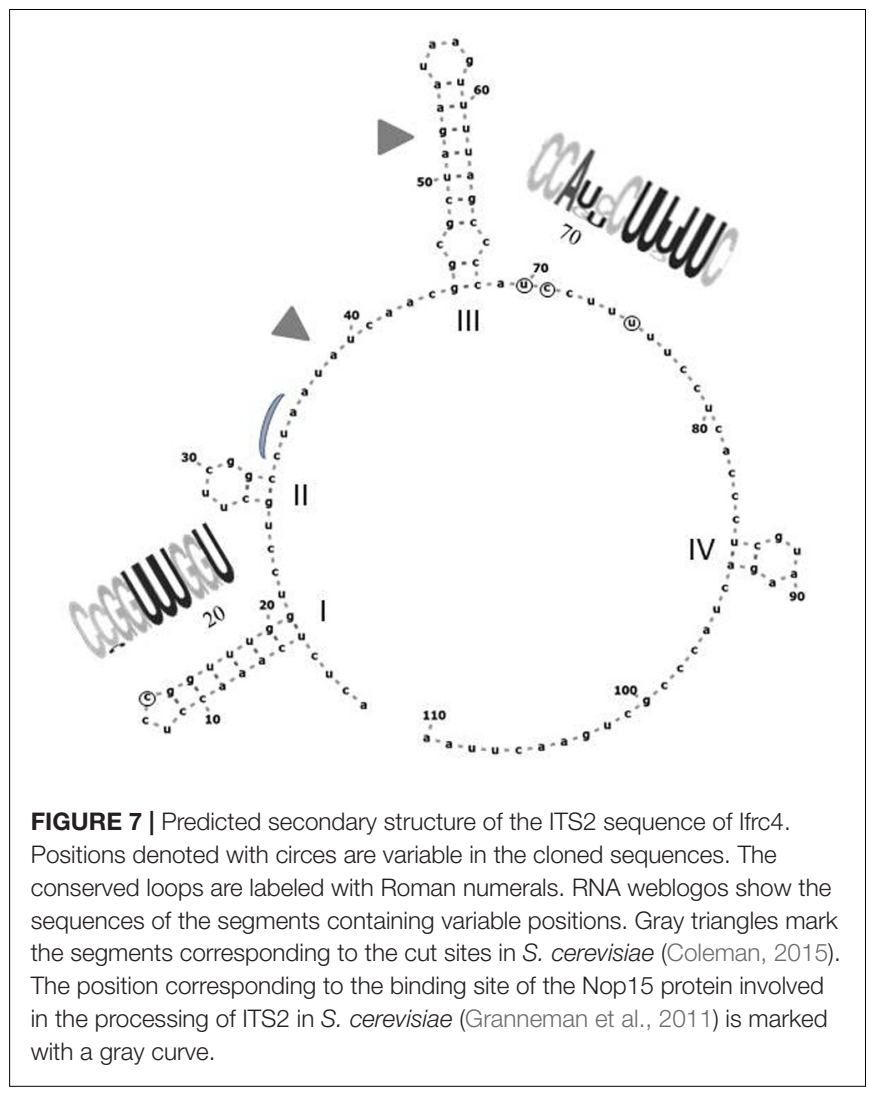

\section{Hybridisation}

The biological isolation of the two strains was tested by hybridisation attempts. For easier identification of the hybrids, auxotrophic mutants were produced from both strains by mutagenesis. The stable mutants of different auxotrophies were mass-mated in all possible combinations. Surprisingly, no prototrophic colonies (putative hybrids) were formed between conspecific mutants. Their inability to form hybrids hinted at the possibility that the $M$. andauensis and $M$. fructicola type strains could be sexually isolated. To test the mating capabilities of the mutants, we tried to cross them with three auxotrophic mutants of the type strain of $M$. pulcherrima. All produced prototrophic colonies at the intersections with the $M$. pulcherrima mutants Mp5 and Mp13 but not with Mp12 (Figure 8). These results imply that the $M$. andauensis and $M$. fructicola mutants were not sterile but sexually incompatible with each other. When the M. pulcherrima mutants were mass-mated with each other, both Mp5 and Mp 13 produced prototrophs with Mp12, but not with each other (Table 3). Hence, the mutants of the three species could be divided in two groups of different mating activities. All $M$. andauensis and $M$. fructicola mutants as well as the $M$. pulcherrima mutant Mp12 formed one group and the M. pulcherrima mutants Mp5 and Mp13 formed a different group. Members of the same group could not hybridize (mate) with each other but could form hybrids with members of the other group (Table 3). This mating behavior

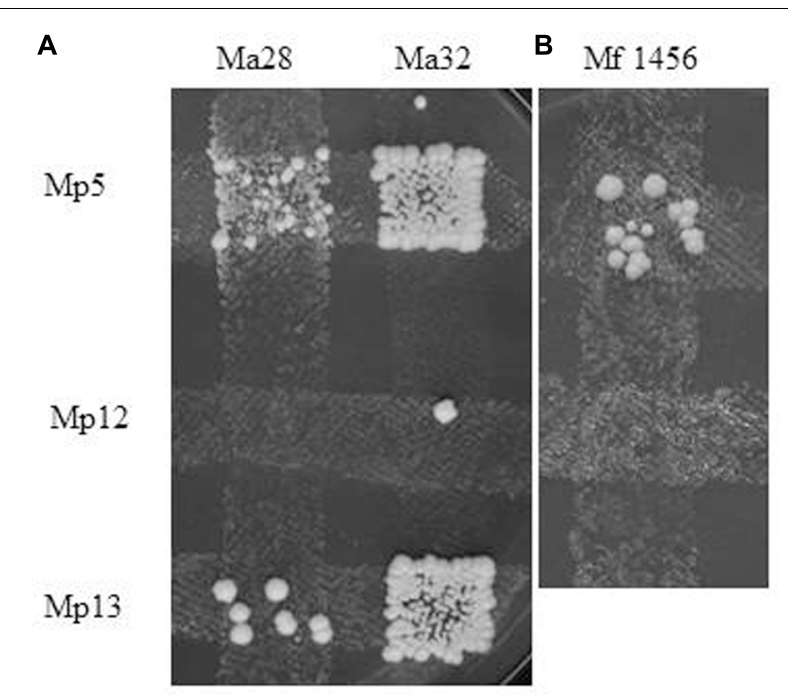

FIGURE 8 | Interspecies hybridisation. Formation of prototrophs at the intersections of $\mathbf{M}$. pulcherrima mutants with (A) $M$. andauensis and (B) M. fructicola mutants of opposite mating type and different auxotrophies. Ma32 is slightly leaky, the two colonies formed outside the intersections with Mp5 and Mp13 can be revertants. The week background growth in the intersection of Ma28 and Mp5 is due to crossfeeding of the former by the latter.

is reminiscent of heterothallic mating types of other yeast species, designated "a" and "alpha" or "plus" and "minus." We will therefore tentatively denote the larger group "a" and the smaller group "alpha." Very rarely prototrophic colonies appeared at the intersections of strains belonging to the same mating type, but their frequency did not exceed the frequency of spontaneous revertants (Figure 8A). The sexual activities of ascomyceteous yeasts are determined by matingtype determinants referred to as MATa and MATalpha in most species. To identify the counterparts of these determinants we searched the $M$. fructicola genome sequence with the Clavispora lusitaniae orthologs (CLUG_04923, CLUG_02322 and CLUG_04271) of the S. cerevisiae proteins MATalpha1, MATalpha2, and HMRa1. The search detected ORFs coding for similar proteins. Thus, $M$. fructicola CBS $8853^{\mathrm{T}}$ possesses counterparts of the central regulators of both mating types of Saccharomyces.

\section{Clamidospore Formation, Sporulation, and Segregation of Hybrids}

Both $M$. andauensis and M. fructicola were described as species producing asci containing needle-shape ascospores (Kurtzman and Droby, 2001; Molnar and Prillinger, 2005) and pulcherrima cells assumed to be the forms from which asci can develop (Pitt and Miller, 1968). We found that all mutant and hybrid cultures contained 5 to $70 \%$ pulcherrima cells when cultivated on vegetable agar at low temperature. We did not observe conjugation between pulcherrima cells or between budding cells. Conjugation was not observed in samples taken from intersections of the hybridisation grids either. The mutants 
TABLE 3 | Hybridisation of auxotrophic mutants and segregants.

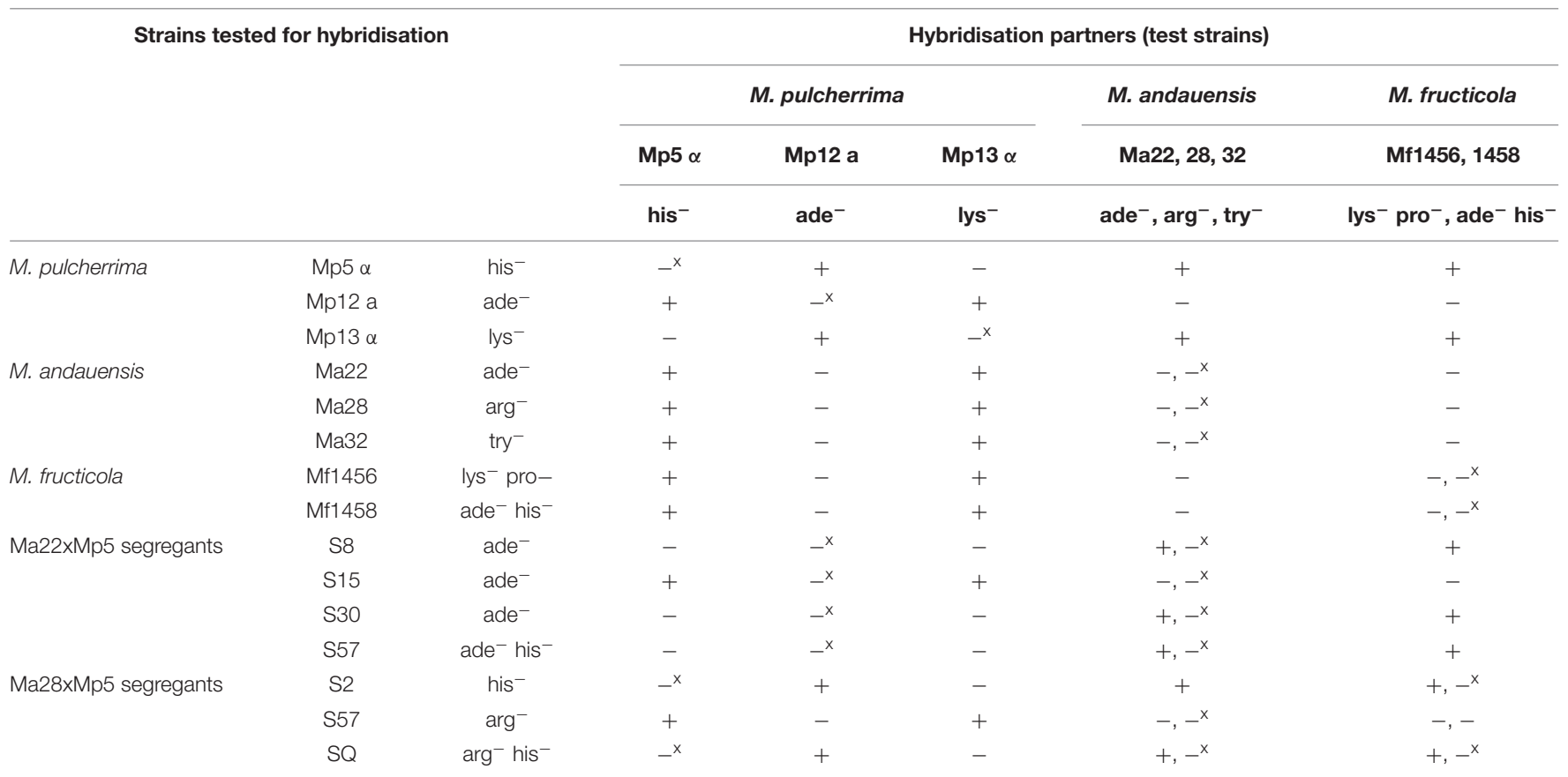

+ , prototrophs are produced at the intersections; $x$, match of identical auxotrophic markers.

formed asci with frequencies $<0.1 \%$ but most asci contained no discernible spores (Figures 9A,B). The $M$. andauensis and $M$. pulcherrima hybrids formed up to $25 \%$ asci which were much larger than the asci of the parental mutants and usually had two spores (Figures 9C,D). Spontaneous ascus autolysis was observed very rarely (Figure 9H). Treatment with the cell-wall lytic enzyme Zymolyase degraded the ascus wall and resulted in free spores (Figures 9I,J). 80 individual intact asci and 70 Zymolyase-released spores were separated by micromanipulation from samples transferred on YEA plates and monitored for 4 weeks at room temperature. None of the spores germinated. No spore germination was observed in hundreds of asci and free spores released from the asci by Zymolyase treatment on YEA films sandwiched between glass slides and cover slips either. In contrast, the pulcherrima cells germinated in the sandwich cultures (Figure 9E) and formed pseudohyphae (Figures 9F,G) which then gradually returned to the standard budding morphology after a couple of cell divisions. DAPI-staining detected fluorescent material in most ascospores similar to that visible in the nuclei of vegetative cells. No germination was observed in samples frozen before spreading on YEA films either. We tested the effect of freezing because pigmented Metschnikowia strains are common mainly in climatic zones with harsh winter conditions.

Although the spores seemed dormant, we checked the sporulating cultures of the hybrids for segregation by spreading their cells on a complete medium to obtain individual single-cell colonies. After replica-plating the colonies on a minimal medium, we found 4 and 14 auxotrophic colonies among 950 colonies of the Ma22 × Mp5 and Ma28 × Mp5 hybrids, respectively
(Table 3). In both cases one of the prototrophs was recombinant showing the auxotrophies of both parents. The segregants were then tested also for mating activity. All formed prototrophic hybrids with mutants of only one or the other mating type. Thus, they were heterothallic like their parental strains, but in some of them the parental marker and the parental mating type separated. For example, all segregants of the Ma22 × Mp5 hybrid were ade ${ }^{-}$but only one showed the mating activity of the $M$. andauensis parental strain. No segregation was detected among 1000 colonies when the $M$. fructicola $\times$ M. pulcherrima hybrids were tested.

\section{DISCUSSION}

In a previous study we found that the type strains of $M$. andauensis and $M$ fructicola had highly heterogeneous LSU (26S) rRNA gene paralogs that formed a continuous pool leaving no barcode gap suitable for differentiation of these two species by D1/D2 sequencing (Sipiczki et al., 2013). In this work we extended the investigations to the ITS1-5.8S-ITS2 regions of their rDNA repeats. We found that like the D1/D2 domains, both ITS spacers of both strains had ambiguous nucleotides when the regions were sequenced directly from the genomes. To resolve the observed sequence ambiguities, we randomly cloned individual DNA molecules from the amplified DNA preparation and determined the sequence of each clone. All $M$. andauensis clones and most $M$. fructicola clones had unique sequences. The revealed polymorphism cannot be attributed to sequencing errors because the variable sites were not scattered randomly across the cloned regions. When we blasted the cloned 


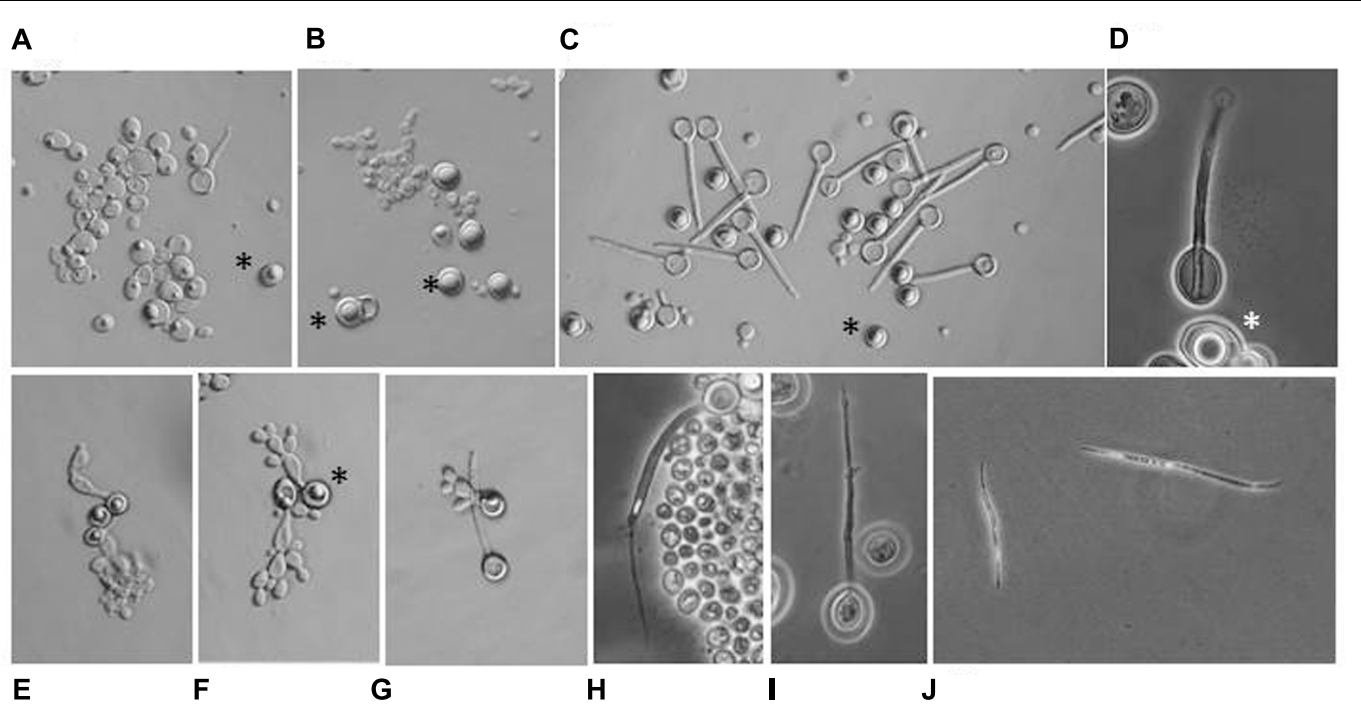

FIGURE 9 | Microscopic morphology. (A) M. andauensis Ma22. Rudimentary asci with no discernable spores can occasionally seen among the cells. (B) M. pulcherrima Mp5. (C) Hybrid Ma22xMp5. (D) A two-spored ascus from the hybrid MA22xMp5. (E) Germinating pulcherrima cell (chlamydospore). (F) Pseudohypha formed by germinating pulcherrima cells. (G) Germination of pulcherrima cells is not accompanied with germination of ascospores. (H) Rare spontaneous release of ascospore. (I) Release of ascospore facilitated by Zymolyase treatment. (J) Free ascospores released by Zymolyase-treated asci. (A-D) Samples taken from cultures incubated on vegetable agar at $12^{\circ} \mathrm{C}$ for 4 weeks. (E-H) Sandwich cultures on YEA films incubated at $20^{\circ} \mathrm{C}$. (E-G) After 72 h. (H) After 7 days. Stars denote pulcherrima cells (chlamydospores).

sequences against the draft genome sequence (Hershkovitz et al., 2013) of the M. fructicola type strain, we found additional sequence versions. Identity with a cloned sequence was found only in one genomic locus. Interestingly, certain $M$. fructicola genomic sequences were more similar to certain $M$. andauensis clones than to their own paralogs. This finding reinforces the notion based on D1/D2 analysis (Sipiczki et al., 2013) that these organisms share a pool of non-homogenized rDNA repeats.

Intragenomic (within-individual) ITS diversity has been detected in a small percentage of fungi. In a recent analysis by pyrosequencing of ITS amplicons of single-spore clones of 44 Ascomycota and 55 Basidiomycota species, Lindner et al. (2013) found significant intragenomic ITS polymorphism in only four species. The diversity in the Metschnikowia strains studied in this work was much bigger (14.7\%) than the intragenomic diversity detected in the genome sequences of filamentous fungi and mushrooms for which data are available (e.g., O'Donnell and Cigelnik, 1997; Ko and Jung, 2002; Kauserud and Schumacher, 2003; Kauserud et al., 2007; Simon and Weiss, 2008; Kovacs et al., 2011; Lindner and Banik, 2011; Vydryakova et al., 2012; Lindner et al., 2013). Intragenomic ITS polymorphism of this extent has not been described in yeasts either, although ITS heterogeneity has been reported for several species. The only yeast species in which comparable polymorphism was revealed is Geotrichum candidum (Alper et al., 2011). The intragenomic diversity (26 variable positions) detected in two G. candidum strains was only slightly lower than the diversity ( 34 polymorphic sites) in our Metschnikowia strains. In the more closely related species Pichia membranifaciens only two types of ITS1 and two types of ITS2 sequences were detected (Wu et al., 2006). Large-scale analysis of rDNA repeats in strains of Ashbya gossypii, Cryptococcus neoformans and two Saccharomyces species (Ganley and Kobayashi, 2007) detected differences among rDNA units but the number of polymorphic positions was very low and, unlike the pattern we report here, the polymorphic sites were scattered randomly throughout the entire repeat units. Zhao et al. (2015) identified four and three polymorphic sites in the ITS sequences of Candida glabrata and P. norvegensis clinical isolates. In a Xanthophyllomyces strain two ITS sequences differing by 2 nucleotides were identified (Fell et al., 2007). The intragenomic diversity of the Metschnikowia sequences determined is this study was so big that some of them turned out to be more different from certain paralogs than from their orthologs in certain taxonomically more distant species. Taking together the intragenomic D1/D2 diversity described previously and the ITS diversity revealed in this study, it can be concluded that rDNA sequencing cannot be used for the taxonomic separation of M. andauensis and M. fructicola from each other and from related species. The high intragenomic diversity may further cause difficulties when yeast communities techniques (e.g., metagenomic analysis) are applied to the investigation of environmental samples and can easily result in misinterpretation of the sequence data. In view of the detected high intragenomic diversity, all strains previously assigned to either of these species (e.g., Piombo et al., 2018) should be taxonomically re-examined. In this context it is worth mentioning that the original taxonomic description of both species was based on rDNA sequences containing numerous ambiguous nucleotides (Kurtzman and Droby, 2001; Molnar and Prillinger, 2005). 
The search of the $M$. fructicola genome sequence for ITS sequences identified $8 \mathrm{rDNA}$ units in 6 sites. One of the sites contained a complete solo rDNA unit, whereas the rest of the sites looked like termini of arrays. As the proximal adjacent sequences of the units differed from each other, it is unlikely that the rDNA of this strain is organized in a single array. Its rDNA units seem to be dispersed in at least five distinct locations, but given the fact that the genome is not completely sequenced and assembled yet (Hershkovitz et al., 2013), the actual number of loci containing rDNA genes may be even higher. In other yeast species examined in detail, the rDNA units are organized in large continuous rDNA arrays and the intragenomic polymorphism is very low due to a process referred to as homogenisation or concerted evolution. In concerted evolution, the rRNA units evolve "horizontally," meaning that a non-harmful mutation that arises in one unit spreads to all other units of the array. As a result, the units of the rDNA array do not evolve independently. The poorly explored underlying mechanism is assumed to involve unequal homologous recombination by sister-chromosome exchange, interchromosomal exchange, intrachromosomal exchange/deletion between rDNA units of the $\operatorname{array}(s)$ (for a review, see Eickbush and Eickbush, 2007) supported by cohesin (to hold broken sister-chromatid ends in place) and the condensation of the rDNA (to ensure proper segregation of the region in the $M$ phase of the cell cycle) (for a review, see Kobayashi, 2006). I has been shown by computer modeling that homogenisation reduces the mutational load (Ohta, 1989). In M. fructicola the rDNA seems to have fragmented organization which may preclude the homogenisation of the physically separated shorter tandems and orphaned copies. This assumption is consistent with the results of a recent study of four Nikotiana species which found correlation between the number of ITS1 ribotypes and the number of locations of rDNA units in the genome (Matyasek et al., 2012). It was proposed that homogenisation can be higher within the arrays than between arrays and the low levels of homogenisation between different chromosomal loci would allow the accumulation of different ribotypes.

Given that the transcripts of both spacers are known to form structures required for correct processing of the transcripts of the rRNA genes (Hillis and Dixon, 1991; Nazar, 2004; Mullineux and Hausner, 2009), the question arises whether all paralogs found in the Metschnikowia strains code for functional ITS transcripts. To answer this question we examined the diversity of the ITS1 and ITS2 secondary structures of all clones and genomic sequences. We found that substitutions at the polymorphic sites of ITS2 regions had no effect on the predicted secondary structure. This structural conservation is consistent with the notion that ITS2 is more strictly conserved than ITS1 in the majority of fungi (e.g., Beiggi and PierceyNormore, 2007; Nilsson et al., 2008) because it is under stronger selective constraints. Typically, this spacer exhibits a low level of sequence variation within species and a high level of divergence between species (for a review see Coleman, 2015). This feature renders the ITS2 sequences useful for taxonomic differentiation and inference of phylogenetic relationships of different taxa. However, in our case the $M$. andauensis and $M$. fructicola ITS2 sequences did not form distinct groups. The ITS1 segments had much more polymorphic sites, and hence the predicted secondary structures of their transcripts showed high level of diversity. It can be assumed that the ITS1-5.8S-ITS2 sequence found in $5 \mathrm{M}$. fructicola clones and one genomic locus might be the "functional standard" in M. fructicola. It was specified as ancestral in the parsimony network analysis and its accompanying D1/D2 sequence was previously found among the cloned D1/D2 domains (Sipiczki et al., 2013). This and the versions differing from it only by a few substitutions with only minor effects on the ITS1 secondary structure may supply the cell with a functional pool of transcripts. Although the positions of the variable sites showed some tendency to accumulate in certain segments, pairs of compensatory substitutions (coevolving pairs of sites) could not be identified. This is a fundamental difference from the D1/D2 domains, in which coevolving pairs of nucleotides in the pairing stretches of loops appeared to safeguard structural stability (Sipiczki et al., 2013). Since the RNA self-splicing activity of ITS1 is also important for the proper assembly of ribosomes (through its role in the maturation of the $18 \mathrm{~S}$ rRNA) (van Nues et al., 1994), the rDNA units encoding ITS1 transcripts of strongly divergent secondary structures might negatively interfere with the "functional standard" in ribosome genesis. This effect can be avoided by silencing and inactivation of these units. Attenuation and silencing preclude or at least hampers the production of aberrant transcripts and thus can protect the cell against the adverse effect of abnormally spliced rRNAs on ribosome formation. At the same time, the activity reduction relieves these rDNA units from the selection pressure, allowing the fixation of all types of mutations with equal probabilities in any part of their sequences regardless of the structural consequences. Accumulation of mutations can gradually degenerate the inactivated rDNA units into non-functional pseudogenes. Pseudogenes are genomic sequences that arise from functional genes but their genetic defects (e.g., mutations) preclude the generation of functional transcripts (for a review, see Balakirev and Ayala, 2003). In plants, non-functional rDNA units containing pseudogenes are recognizable by their irregular $5.8 \mathrm{~S}$ sequences and the absence of some of the ITS regions (Queiroz et al., 2011). We found this kind of truncate structures in two unitigs of the $M$. fructicola genome sequence; both lacked ITS1 and the nucleotide sequences of their $5.8 \mathrm{~S}$ genes were very different from those of the other rDNA units. We assume that these rDNA units may be remnants of erstwhile active units being in an advanced stage of decay progressing in a way analogous to that observed e.g., in Buchnera (Degnan et al., 2011). rDNA pseudogenes occur in high numbers in certain plants (e.g., Bailey et al., 2003; Harpke and Peterson, 2007) but were detected only in few filamentous fungi (e.g., Rooney and Ward, 2005; Lindner and Banik, 2011; Lindner et al., 2013; Li et al., 2017), all being unrelated to Metschnikowia. The dispersed locations of rDNA units in the genome and the presence of truncated copies (pseudogenisation) indicate that the sequence homogeneity of the ITS1-5.8S-ITS2 region of 
M. fructicola is not maintained by homogenisation, and that its rDNA units evolve under a birth-and-death process. In the latter mode of evolution it is selection, not homogenisation that maintains the rDNA units as a coherent family. New copies of genes are created by gene duplications and some duplicated genes are preserved in the genome for long periods of time, whereas others become non-functional through the accumulation of harmful mutations and undergo gradual degradation (for a review, see Nei and Rooney, 2005). The high divergence among the sequences cloned from the $M$. andauensis strain implies that its rDNA is also shaped by birth-anddeath evolution. Nevertheless, the rDNA units corresponding to cloned sequences with divergent secondary structures can only be in early phases of pseudogenisation because they still have complete ITS segments and their $5.8 \mathrm{~S}$ genes differ from each other only at one site. To our knowledge this is the first report of birth-end-death evolution of rDNA in yeasts.

The results of the phylogenetic analysis of the D1/D2 domains of these two species suggested that their non-homogenized 26S rRNA genes had not evolved in a vertical tree-like way but most probably by reticulate intra- and interspecies interactions (Sipiczki et al., 2013). The findings of this study demonstrate that the ITS regions of the repeats are also involved in the reticulation. Neither the statistical parsimony network analysis nor the phylogenetic network analyses grouped the sequences of the strains in distinct clades. Their intermixing in both types of networks indicated that the rDNA units of these strains must have evolved in interaction. For genetic interactions the units of the strains need to be brought together and then separated. As horizontal gene transfer by mechanisms widespread in bacteria is unlikely to take place in Metschnikowia, hybridisation can be assumed to be the major mechanism of bringing the two sets of rDNA units together. Both strains form ascospores (Kurtzman and Droby, 2001; Molnar and Prillinger, 2005) suggesting that both possess sexual phases, in which they can hybridize, provided they are sexually compatible.

In an attempt to test them for sexual compatibility, we generated mutants with complementary auxotrophies from their cultures and co-cultivated them in all possible pair-wise combinations. Unexpectedly, no prototrophs were produced in any combinations as if the mutants were sterile. To find out whether the failure was due to sterility, we crossed them also with three mutants of the related species M. pulcherrima. All $M$. andauensis and M. fructicola mutants formed prototrophs with two M. pulcherrima mutants but not with the third mutant. Hence, neither the M. andauensis nor the M. fructicola mutants were sterile. The two M. pulcherrima mutants compatible with them did not form hybrids with each other but did with the third mutant. These finding implies that all mutants of the three species were heterothallic, and that all $M$. andauensis and $M$. fructicola mutants as well as the M. pulcherrima mutant not hybridizing with them had identical mating type. The inability of their mutants to hybridize with each other, however, does not necessarily mean that $M$. andauensis and M. fructicola are sexually isolated. Both produce asci in pure cultures, albeit at low frequencies, and preliminary results suggested that $M$. fructicola be diploid and heterozygous at the mating type locus (Lachance, 2016). We found potential orthologs of both MATa and MATalpha ORFs in its genome sequence, but their simultaneous presence does not prove that the strain is heterozygous diploid. In numerous other yeast species, heterothallic haploids can have both mating-type determinants, but usually only one is active (e.g., Haber, 2012; Watanabe et al., 2013). Thus, we most probably had heterothallic haploid mutants of identical mating types, indicating that the type strains analyzed may not be homothallic and their cultures may also contain mating-proficient haploid cells. Interestingly, all mutants of the three species formed "pulcherrima cells" (chlamydospores). It was previously proposed that diploid cells develop into chlamydospores (Lachance, 2016). Their abundance in the mutant cultures implies that either haploids can also convert to chlamydospores or the heterothallic haploid cells frequently double their genomes by autodiploidisation. The low sporulation efficiency in the wild-type cultures also argues for the predominance of haploidy because the hybrids with the M. pulcherrima mutants showed very high sporulation activity. Somewhat unexpectedly, the spores of the hybrids did not germinate. This can be attributed to a postzygotic sterility barrier analogous to that operating in the allodiploid hybrids of Saccharomyces species isolated by a double sterility barrier (e.g., Pfliegler et al., 2012). Alternatively, the failure of the ascospores to germinate might be due endogenous inhibitors keeping the spores "dormant." Certain fungi were found to produce "endogenously dormant spores" whose dormancy can be broken by aging or by some physiological shock permitting the endogenous inhibitors to leach out or degrade (e.g., Graham et al., 2014). We tested only one potential shock. Freezing and thawing did not activate the spores.

Nevertheless, the isolation is not absolute because segregants with parental markers and marker combinations were found at low frequencies in the sporulating cultures of the hybrids. It is not clear whether these segregants were clones produced by a few germinating spores or products of mitotic segregation. The latter possibility would be consistent with the results of a recent reinterpretation of some early observations that suggest that $M$. pulcherrima may have a parasexual life cycle (Naumov, 2011). In the model proposed by Naumov (2011), both mitotic (parasexual) and meiotic (sexual) haploidisation can take place in the life cycle of Metschnikowia sensu stricto. Although only indirectly, through the involvement of a third species, the hybridisation experiments prove that the type strains of $M$. andauensis and $M$. fructicola are not completely isolated and hence interspecies (intergenomic) reticulation-type interactions can be involved in the evolution of their rDNA genes. It is worth mentioning here that James et al. (2009) noted that in Saccharomyces many single nucleotide polymorphisms (SNPs) were more common in strains with mosaic/hybrid genomes than in strains with standard genomes, suggesting that hybridization plays a role in the evolution of intragenomic variation even in yeasts having large rDNA arrays suitable for homogenisation. 


\section{AUTHOR CONTRIBUTIONS}

MS conceived and designed the experiments, performed the bioinformatics analysis, and wrote the manuscript. MS, EH, and WP performed the experiments. All authors reviewed the manuscript.

\section{FUNDING}

This study was supported in part by the grant K-124417 provided by the National Research, Development and Innovation Office of Hungary. WP was supported by the European Union and the State of Hungary grant, cofinanced by the European Social Fund in the framework

\section{REFERENCES}

Alper, I., Frenette, M., and Labrie, S. (2011). Ribosomal DNA polymorphisms in the yeast Geotrichum candidum. Fungal Biol. 115, 1259-1269. doi: 10.1016/j.funbio. 2011.09.002

Ankenbrand, M. J., Keller, A., Wolf, M., Schultz, J., and Förster, F. (2015). ITS2 Database V: twice as much. Mol. Biol. Evol. 32, 3030-3032. doi: 10.1093/molbev/ msv174

Bailey, C. D., Carr, T. G., Harris, S. A., and Hughes, C. E. (2003). Characterization of angiosperm nrDNA polymorphism, paralogy, and pseudogenes. Mol. Phylogenet. Evol. 29, 435-455. doi: 10.1016/j.ympev.2003.08.021

Balakirev, E. S., and Ayala, F. J. (2003). Pseudogenes: are they 'junk' or functional DNA? Annu. Rev. Genet. 37, 123-151. doi: 10.1146/annurev.genet.37.040103. 103949

Beiggi, S., and Piercey-Normore, M. D. (2007). Evolution of ITS ribosomal RNA secondary structures in fungal and algal symbionts of selected species of Cladonia sect. Cladonia (Cladoniaceae, Ascomycotina). J. Mol. Evol. 64, 528-542. doi: 10.1007/s00239-006-0115-x

Bruen, T., Philippe, H., and Bryant, D. (2006). A quick and robust statistical test to detect the presence of recombination. Genetics 172, 2665-2681. doi: 10.1534/ genetics.105.048975

Bryant, D., and Moulton, V. (2004). Neighbor-Net: an agglomerative method for the construction of phylogenetic networks. Mol. Biol. Evol. 21, 255-265. doi: $10.1093 / \mathrm{molbev} / \mathrm{msh} 018$

Caetano-Anolle's, G. (2002). Tracing the evolution of RNA structures in ribosomes. Nucleic Acids Res. 30, 2575-2587. doi: 10.1093/nar/30.11.2575

Clement, M., Posada, D., and Crandall, K. A. (2000). TCS: a computer program to estimate gene genealogies. Mol. Ecol. 9, 1657-1660. doi: 10.1046/j.1365-294x. 2000.01020.x

Coleman, A. W. (2015). Nuclear rRNA transcript processing versus internal transcribed spacer secondary structure. Trends Genet. 31, 157-163. doi: 10.1016/j.tig.2015.01.002

Cook, A. H., and Slater, C. A. (1956). The structure of pulcherrimin. J. Chem. Soc. 4133-4135. doi: 10.1039/jr9560004133

Cryle, M. J., Bell, S. G., and Schlichting, I. (2010). Structural and biochemical characterization of the cytochrome P450 CypX (CYP134A1) from Bacillus subtilis: a cyclo-L-leucyl-L-leucyl dipeptide oxidase. Biochemistry 49, 7282-7296. doi: 10.1021/bi100910y

Degnan, P. H., Ochman, H., and Moran, N. A. (2011). Sequence conservation and functional constraint on intergenic spacers in reduced genomes of the obligate symbiont Buchnera. PLoS Genet. 7:e1002252. doi: 10.1371/journal. pgen. 1002252

Eickbush, T. H., and Eickbush, D. G. (2007). Finely orchestrated movements: evolution of the ribosomal RNA genes. Genetics 175, 477-485. doi: 10.1534/ genetics.107.071399

Elder, J. F., and Turner, B. J. (1995). Concerted evolution of repetitive DNA sequences in eukaryotes. Q. Rev. Biol. 70, 297-320. doi: 10.1086/419073 of TÁMOP-4.2.4.A/2-11/1-2012-0001 "National Excellence Program.”

\section{ACKNOWLEDGMENTS}

The authors thank Anita Olah-Csabai for expert technical assistance.

\section{SUPPLEMENTARY MATERIAL}

The Supplementary Material for this article can be found online at: https://www.frontiersin.org/articles/10.3389/fmicb. 2018.01193/full\#supplementary-material

Fell, J. W., Scorzetti, G., Statzell-Tallman, A., and Boundy-Mills, K. (2007). Molecular diversity and intragenomic variability in the yeast genus Xanthophyllomyces: the origin of Phaffia rhodozyma? FEMS Yeast Res. 7, 1399-1408.

Ganley, A. R., and Kobayashi, T. (2007). Highly efficient concerted evolution in the ribosomal DNA repeats: total rDNA repeat variation revealed by whole-genome shotgun sequence data. Genome Res. 17, 184-191. doi: 10.1101/gr.5457707

Gorodkin, J., Heyer, L. J., Brunak, S., and Stormo, G. D. (1997). Displaying the information contents of structural RNA alignments: the structure logos. Comput. Appl. Biosci. 13, 583-586.

Graham, J. K., Smith, M. L., and Simons, A. M. (2014). Experimental evolution of bet hedging under manipulated environmental uncertainty in Neurospora crassa. Proc. R. Soc. B Biol. Sci. 281:20140706. doi: 10.1098/rspb.2014.0706

Granneman, S., Petfalski, E., and Tollervey, D. (2011). A cluster of ribosome synthesis factors regulate pre-rRNA folding and 5.8S rRNA maturation by the Rat1 exonuclease. EMBO J. 19, 4006-4019. doi: 10.1038/emboj.2011.256

Guzmán, B., Lachance, M. A., and Herrera, C. M. (2013). Phylogenetic analysis of the angiosperm-floricolous insect-yeast association: have yeast and angiosperm lineages co-diversified? Mol. Phylogenet. Evol. 68, 161-175. doi: 10.1016/j. ympev.2013.04.003

Haber, J. E. (2012). Mating-type genes and MAT switching in Saccharomyces cerevisiae. Genetics 191, 33-64. doi: 10.1534/genetics.111.134577

Harpke, D., and Peterson, A. (2007). Quantitative PCR revealed a minority of ITS copies to be functional in Mammillaria (Cactaceae). Int. J. Plant Sci. 168, 1157-1160. doi: 10.1086/520729

Hasegawa, M., Kishino, H., and Yano, T. (1985). Dating of human-ape splitting by a molecular clock of mitochondrial DNA. J. Mol. Evol. 22, 160-174. doi: 10.1007/BF02101694

Hershkovitz, V., Sela, N., Taha-Salaime, L., Liu, J., Rafael, G., Kessler, C., et al. (2013). De-novo assembly and characterization of the transcriptome of Metschnikowia fructicola reveals differences in gene expression following interaction with Penicillium digitatum and grapefruit peel. BMC Genomics 14:168. doi: 10.1186/1471-2164-14-168

Hillis, D. M., and Dixon, M. T. (1991). Ribosomal DNA: molecular evolution and phylogenetic inference. Q. Rev. Biol. 66, 411-453. doi: 10.1086/417338

James, S. A., O’Kelly, M. J. T., Carter, D. M., Davey, R. P., van Oudenaarden, A., and Roberts, I. N. (2009). Repetitive sequence variation and dynamics in the ribosomal DNA array of Saccharomyces cerevisiae as revealed by whole-genome resequencing. Genome Res. 19, 626-635. doi: 10.1101/gr.084517.108

Kauserud, H., and Schumacher, T. (2003). Ribosomal DNA variation, recombination and inheritance in the basidiomycete Trichaptum abietinum: implications for reticulate evolution. Heredity 91, 163-172. doi: 10.1038/sj.hdy.6800294

Kauserud, H., Vegården, I. B., Decock, C., and Hallenberg, N. (2007). Hybridization among cryptic species of the cellar fungus Coniophora puteana (Basidiomycota). Mol. Ecol. 16, 389-399. doi: 10.1111/j.1365-294X.2006. 03129.x 
Kerpedjiev, P., Hammer, S., and Hofacker, I. L. (2015). Forna (force-directed RNA): simple and effective online RNA secondary structure diagrams. Bioinformatics 31, 3377-3379. doi: 10.1093/bioinformatics/btv372

Kluyver, A. J., van der Walt, J. P., and van Triet, A. J. (1953). Pulcherrimin, the pigment of Candida pulcherrima. Proc. Natl. Acad. Sci. U.S.A. 39, 583-593. doi: 10.1073/pnas.39.7.583

Ko, K. S., and Jung, H. S. (2002). Three nonorthologous ITS1 types are present in a polypore fungus Trichaptum abietinum. Mol. Phylogenet. Evol. 23, 112-122. doi: 10.1016/S1055-7903(02)00009-X

Kobayashi, T. (2006). Strategies to maintain the stability of the ribosomal RNA gene repeats-collaboration of recombination, cohesion, and condensation. Genes Genet. Syst. 81, 155-161. doi: 10.1266/ggs.81.155

Kovacs, G. M., Balazs, T. K., Calonge, F. D., and Martín, M. P. (2011). The diversity of Terfezia desert truffles: new species and a highly variable species complex with intrasporocarpic nrDNA ITS heterogeneity. Mycologia 103, 841-853. doi: $10.3852 / 10-312$

Kurtzman, C. P., and Droby, S. (2001). Metschnikowia fructicola, a new ascosporic yeast with potential for biocontrol of postharvest fruit rots. Syst. Appl. Microbiol. 24, 395-399. doi: 10.1078/0723-2020-00045

Kurtzman, C. P., Robnett, C. J., and Basehoar, E. (2018). Four new species of the yeast genus Metschnikowia and the transfer of seven Candida species to Metschnikowia and Clavispora as new combinations. Antonie Van Leeuwenhoek doi: 10.1007/s10482-018-1095-8 [Epub ahead of print]. doi: 10.1007/s10482018-1095-8

Lachance, M. A. (2011). "Metschnikowia Kamienski (1899)," in The Yeasts. A Taxonomic Study, eds C. P. Kurtzman, J. W. Fell, and T. Boekhout (Amsterdam: Elsevier), 575-620.

Lachance, M. A. (2016). Metschnikowia: half tetrads, a regicide and the fountain of youth. Yeast 33, 563-574. doi: 10.1002/yea.3208

Li, Y., Yang, R. H., Jiang, L., Hu, X. D., Wu, Z. J., and Yao, Y. J. (2017). rRNA Pseudogenes in filamentous ascomycetes as revealed by genome data. G3 7, 2695-2703. doi: 10.1534/g3.117.044016

Lindner, D. L., and Banik, M. T. (2011). Intragenomic variation in the ITS rDNA region obscures phylogenetic relationships and inflates estimates of operational taxonomic units in genus Laetiporus. Mycologia 103, 731-740. doi: 10.3852/ 10-331

Lindner, D. L., Carlsen, T., Nilsson, R. H., Davey, M., Schumacher, T., and Kauserud, H. (2013). Employing 454 amplicon pyrosequencing to reveal intragenomic divergence in the internal transcribed spacer rDNA region in fungi. Ecol. Evol. 3, 1751-1764. doi: 10.1002/ece3.586

Markham, N. R., and Zuker, M. (2008). UNAFold: software for nucleic acid folding and hybridization. Methods Mol. Biol. 453, 3-31. doi: 10.1007/978-1-60327429-6_1

Matyasek, R., Renny-Byfield, S., Fulnecek, J., Macas, J., Grandbastien, M. A., Nichols, R., et al. (2012). Next generation sequencing analysis reveals a relationship between rDNA unit diversity and locus number in Nicotiana diploids. BMC Genomics 13:722. doi: 10.1186/1471-2164-13-722

Molnar, O., and Prillinger, H. (2005). Analysis of yeast isolates related to Metschnikowia pulcherrima using the partial sequence of the large subunit rDNA and the actin gene; description of Metschnikowia andauensi sp. Syst. Appl. Microbiol. 28, 717-726. doi: 10.1016/j.syapm.2005. 05.009

Mullineux, T., and Hausner, G. (2009). Evolution of rDNA ITS1 and ITS2 sequences and RNA secondary structures within members of the fungal genera Grosmannia and Leptographium. Fungal Genet. Biol. 46, 855-867. doi: 10.1016/ j.fgb.2009.08.001

Naumov, G. I. (2011). Molecular and genetic differentiation of small-spored species of the yeast genus Metschnikowia Kamienski. Mikrobiologiya 80, 147-154. doi: 10.1134/S0026261711020111

Nazar, R. N. (2004). Ribosomal RNA processing and ribosome biogenesis in eukaryotes. IUBMB Life 56, 457-465. doi: 10.1080/15216540400010867

Nei, M., and Rooney, A. P. (2005). Concerted and birth-and-death evolution of multigene families. Annu. Rev. Genet. 39, 121-152. doi: 10.1146/annurev.genet. 39.073003.112240

Nilsson, R. H., Kristiansson, E., Ryberg, M., Hallenberg, N., and Larsson, K.-H. (2008). Intraspecific ITS variability in the kingdom Fungi as expressed in the international sequence databases and its implications for molecular species identification. Evol. Bioinformatics 4, 193-201. doi: 10.4137/EBO.S653
O’Donnell, K., and Cigelnik, E. (1997). Two divergent intragenomic rDNA ITS2 types within a monophyletic lineage of the fungus Fusarium are nonorthologous. Mol. Phylogenet. Evol. 7, 103-116. doi: 10.1006/mpev.1996. 0376

Ohta, T. (1989). The mutational load of a multigene family with uniform members. Genet. Res. 53, 141-145. doi: 10.1017/S0016672300028020

Pfliegler, W. P., Antunovics, Z., and Sipiczki, M. (2012). Double sterility barrier between Saccharomyces species and its breakdown in allopolyploid hybrids by chromosome loss. FEMS Yeast Res. 12, 703-718. doi: 10.1111/j.1567-1364.2012. 00820.x

Piombo, E., Sela, N., Wisniewski, M., Hoffmann, M., Gullino, M. L., Allard, M. W., et al. (2018). Genome sequence, assembly and characterization of two Metschnikowia fructicola strains used as biocontrol agents of postharvest diseases. Front. Microbiol. 9:593. doi: 10.3389/fmicb.2018.00593

Pitt, J. I., and Miller, M. W. (1968). Sporulation in Candida pulcherrima, Candida reukaufii and Chlamydozyma species: their relationships with Metschnikowia. Mycologia 60, 663-685. doi: 10.2307/3757434

Queiroz, C. S., Batista, F. R. C., and Oliveira, L. O. (2011). Evolution of the 5.8S nrDNA gene and internal transcribed spacers in Carapichea ipecacuanha (Rubiaceae) within a phylogeographic context. Mol. Phylogenet. Evol. 59, 293-302. doi: 10.1016/j.ympev.2011.01.013

Rooney, A. P., and Ward, T. J. (2005). Evolution of a large ribosomal RNA multigene family in filamentous fungi: birth and death of a concerted evolution paradigm. Proc. Natl. Acad. Sci. U.S.A. 102, 5084-5089. doi: 10.1073/pnas. 0409689102

Schlotterer, C., Hauser, M.-T., von Haeseler, A., and Tautz, D. (1994). Comparative evolutionary analysis of rDNA ITS regions in Drosophila. Mol. Biol. Evol. 11, 513-522.

Schoch, C. L., Seifert, K. A., Huhndorf, A., Robert, V., Spouge, J. L., Levesque, C. A., et al. (2012). Nuclear ribosomal internal transcribed spacer (ITS) region as a universal DNA barcode marker for Fungi. Proc. Natl. Acad. Sci. U.S.A. 109, 6241-6246. doi: 10.1073/pnas.1117018109

Schultz, J., Maisel, S., Gerlach, D., Müller, T., and Wolf, M. (2005). A common core of secondary structure of the internal transcribed spacer 2 (ITS2) throughout the Eukaryota. RNA 11, 361-364. doi: 10.1261/rna.7204505

Simon, U. K., and Weiss, M. (2008). Intragenomic variation of fungal ribosomal genes is higher than previously thought. Mol. Biol. Evol. 25, 2251-2254. doi: $10.1093 / \mathrm{molbev} / \mathrm{msn} 188$

Sipiczki, M. (2003). Candida zemplinina sp. nov., an osmotolerant and psychrotolerant yeast that ferments sweet botrytized wines. Int. J. Syst. Evol. Microbiol. 53, 2079-2083. doi: 10.1099/ijs.0.02649-0

Sipiczki, M. (2006). Metschnikowia strains isolated from botrytized grapes antagonize fungal and bacterial growth by iron depletion. Appl. Environ. Microbiol. 72, 6716-6724. doi: 10.1128/AEM.01275-06

Sipiczki, M. (2012). Pichia bruneiensis sp. nov., a biofilm-producing dimorphic yeast species isolated from flowers in Borneo. Int. J. Syst. Evol. Microbiol. 62, 3099-3104. doi: 10.1099/ijs.0.044974-0

Sipiczki, M., and Ferenczy, L. (1978). Enzymic methods for enrichment of fungal mutants I. Enrichment of Schizosaccharomyces pombe mutants. Mutat. Res. 50, 163-173. doi: 10.1016/0027-5107(78)90021-0

Sipiczki, M., Pfliegler, W. P., and Holb, I. J. (2013). Metschnikowia species share a pool of diverse rRNA genes differing in regions that determine hairpin-loop structures and evolve by reticulation. PLoS One 8:e67384. doi: 10.1371/journal. pone.0067384

Sipiczki, M., Takeo, K., Yamaguchi, M., Yoshida, S., and Miklos, I. (1998). Environmentally controlled dimorphic cycle in a fission yeast. Microbiology 144, 1319-1330. doi: 10.1099/00221287-144-5-1319

Thompson, J. D., Higgions, D. G., and Gibson, T. J. (1994). CLUSTALW: improving the sensitivity of progressive multiple sequence alignment through sequence weighting, position-specific gap penalties and weight matrix choice. Nucleic Acids Res. 22, 4673-4680. doi: 10.1093/nar/22.22.4673

Torres-Machorro, A. L., Hernandez, R., Cevallos, A. M., and Lopez-Villasenor, I. (2010). Ribosomal RNA genes in eukaryotic microorganisms: witnesses of phylogeny? FEMS Microbiol. Rev. 34, 59-86. doi: 10.1111/j.1574-6976.2009. 00196.x

van Nues, R. W., Rientjes, J. M. J., van der Sande, C. A. F. M., Zerp, S. F., Sluiter, C., Venema, J., et al. (1994). Separate structural elements within internal transcribed spacer 1 of Saccharomyces cerevisiae precursor ribosomal RNA 
direct the formation of $17 \mathrm{~S}$ and 26S rRNA. Nucleic Acids Res. 22, 912-919. doi: $10.1093 /$ nar/22.6.912

Venema, J., and Tollervey, D. (1999). Ribosome synthesis in Saccharomyces cerevisiae. Annu. Rev. Genet. 33, 261-311. doi: 10.1146/annurev.genet.33.1.261

Vydryakova, G. A., Van, D. T., Shoukouhi, P., Psurtseva, N. V., and Bissett, J. (2012). Intergenomic and intragenomic ITS sequence heterogeneity in Neonothopanus nambi (Agaricales) from Vietnam. Mycology 3, 89-99.

Watanabe, J., Uehara, K., and Mogi, Y. (2013). Diversity of mating-type chromosome structures in the yeast Zygosaccharomyces rouxii caused by ectopic exchanges between MAT-like loci. PLoS One 8:e62121. doi: 10.1371/journal. pone. 0062121

White, T. J., Bruns, T., Lee, S., and Taylor, J. (1990). "Amplification and sequencing of fungal ribosomal RNA genes for phylogenetics," in PCR Protocols. A Guide to Methods and Applications, eds M. A. Innis, D. H. Gelfand, J. J. Sninsky, and T. J. White (San Diego, CA: Academic Press), 315-322.

Wolf, M., Achtziger, M., Schultz, J., Dandekar, T., and Müller, T. (2005). Homology modeling revealed more than 20,000 rRNA internal transcribed spacer 2 (ITS2) secondary structures. RNA 11, 1616-1623. doi: 10.1261/rna.2144205

Wu, Z. W., Robert, V., and Bai, F. Y. (2006). Genetic diversity of the Pichia membranifaciens strains revealed from rRNA gene sequencing and electrophoretic karyotyping, and the proposal of Candida californica comb. nov. FEMS Yeast Res. 6, 305-311. doi: 10.1111/j.1567-1364.2006.00029.x

Xue, M. L., Zhang, L. Q., Wang, Q. M., Zhang, J. S., and Bai, F. Y. (2006). Metschnikowia sinensis sp. nov., Metschnikowia zizyphicola sp. nov. and
Metschnikowia shanxiensis sp. nov., novel yeast species from jujube fruit. Int. J. Syst. Evol. Microbiol. 56, 2245-2250. doi: 10.1099/ijs.0.64391-0

Zhao, Y., Tsang, C. C., Xiao, M., Cheng, J., Xu, Y., Lau, S. K., et al. (2015). Intra-genomic internal transcribed spacer region sequence heterogeneity and molecular diagnosis in clinical microbiology. Int. J. Mol. Sci. 16, 25067-25079. doi: 10.3390/ijms161025067

Zimmer, E. A., Martin, S. L., Beverley, S. M., Kan, Y. W., and Wilson, A. C. (1980). Rapid duplication and loss of genes coding for the chains of hemoglobin. Proc. Natl. Acad. Sci. U.S.A. 77, 2158-2162. doi: 10.1073/pnas.77.4.2158

Zuker, M. (2003). Mfold web server for nucleic acid folding and hybridization prediction. Nucleic Acids Res. 31, 3406-3415. doi: 10.1093/nar/ gkg595

Conflict of Interest Statement: The authors declare that the research was conducted in the absence of any commercial or financial relationships that could be construed as a potential conflict of interest.

Copyright (c) 2018 Sipiczki, Horvath and Pfliegler. This is an open-access article distributed under the terms of the Creative Commons Attribution License (CC BY). The use, distribution or reproduction in other forums is permitted, provided the original author(s) and the copyright owner are credited and that the original publication in this journal is cited, in accordance with accepted academic practice. No use, distribution or reproduction is permitted which does not comply with these terms. 